\title{
DIFFUSION ON SURFACES AND THE BOUNDARY PERIODIC UNFOLDING OPERATOR WITH AN APPLICATION TO CARCINOGENESIS IN HUMAN CELLS*
}

\author{
ISABELL GRAF ${ }^{\dagger}$ AND MALTE A. PETER ${ }^{\ddagger}$
}

\begin{abstract}
In the context of periodic homogenization based on the periodic unfolding method, we extend the existing convergence results for the boundary periodic unfolding operator to gradients defined on manifolds. These general results are then used to homogenize a system of five coupled reaction-diffusion equations, three of which are defined on a manifold. The system describes the carcinogenesis of a human cell caused by Benzo-[a]-pyrene molecules. These molecules are activated to carcinogens in a series of chemical reactions at the surface of the endoplasmic reticulum. The diffusion on the endoplasmic reticulum, modeled as a Riemannian manifold, is described by the Laplace-Beltrami operator. The binding process to the surface of the endoplasmic reticulum is modeled in a nonlinear way taking into account the number of free receptors.
\end{abstract}

Key words. periodic homogenization, periodic unfolding method, carcinogenesis, reactiondiffusion system, surface diffusion

AMS subject classifications. 35B27, 35K51, 35K58, 92C37

DOI. $10.1137 / 130921015$

1. Introduction. Periodic homogenization is a method for upscaling rigorously mathematical models of multiscale processes. In many cases, the multiscale nature of the problem is due to a microstructure of the material under consideration. While it is infeasible to resolve the microstructure in detail in numerical simulations (and often unnecessary), upscaled models describing the processes on an observation scale much larger than the characteristic size of the microstructure are required. In periodic homogenization, such upscaled models are obtained by assuming the microstructure of the material to be periodic with respect to a reference cell and considering the limit as the periodicity length $\varepsilon>0$ approaches zero. Monographs on the subject include $[3,28,24,21,8,22]$.

An elegant technique for performing periodic homogenization is the periodic unfolding method developed in $[9,7,6,11,5]$. In these articles, many assertions, which are useful for homogenizing partial differential equations, are proved, for instance, convergence results for the periodic unfolding operator involving gradients defined in the domain (summarized in Theorem 2.2) and basic properties of the boundary periodic unfolding operator (Lemma 2.4).

We extend the theory of the periodic unfolding operator acting on hypersurfaces to results for gradients of functions defined on a smooth periodic manifold. In Lemma 2.5 , the weak convergence of a product of functions on a periodic manifold is stated. A connection between the gradient with respect to $\Gamma_{\varepsilon}$ and with respect to $\Gamma$ is deduced in Lemma 2.6. In Theorem 2.9 we give a convergence result for gradients defined on manifolds.

* Received by the editors May 14, 2013; accepted for publication (in revised form) May 20, 2014; published electronically August 28, 2014.

http://www.siam.org/journals/sima/46-4/92101.html

$\dagger$ Institute of Mathematics, University of Augsburg, 86135 Augsburg, Germany. Current address: Department of Mathematics, Simon Fraser University, Burnaby BC, Canada (imgraf@sfu.ca).

$\ddagger$ Institute of Mathematics, University of Augsburg, 86135 Augsburg, Germany and Augsburg Centre for Innovative Technologies, 86135 Augsburg, Germany (malte.peter@math.uni-augsburg.de). 
In the second part of the paper, we apply the general results to homogenize a model for carcinogenesis of a human cell, where carcinogenic molecules invade a cell, undergo chemical reactions to more aggressive molecules and enter the nucleus to bind to the DNA. The transformations to the aggressive molecules happen at the surface of the endoplasmic reticulum. Binding to the surface of the endoplasmic reticulum works as connecting to receptors, which are part of the endoplasmic reticulum. Natural cleaning mechanisms render the carcinogenic molecules harmless. These cleaning enzymes mainly occur in the cytosol. We refer to [13, 27, 30, 25] for this information and further details on the subject.

This mechanism is modeled by a system of five coupled partial differential equations. We emphasize the binding process to the endoplasmic reticulum by including a function that describes the relative concentration of free receptors. To bind to the surface, molecules need to find a free receptor [14], which is modeled by the product of concentration of molecules and receptors based on the law of mass action. This product makes the binding term nonlinear. Other nonlinear terms are the cleaning of molecules in cytosol and the transformations of the molecules on the surface. Much simpler carcinogenesis models taking into account the main subprocesses are found in $[4,16]$.

The endoplasmic reticulum is a bilayered membrane, which pervades the whole cytoplasm of the cell; cf. [14]. One can assume that, roughly speaking, the endoplasmic reticulum is everywhere and nowhere in the cell. To handle this fine structure, we use periodic homogenization based on the periodic unfolding method, which requires the use of results of the first part of this article.

As the biochemical processes in the cell contributing to carcinogenesis take place on the microscopic scale, it is expected that multiscale models taking into account this microstructure, such as the one developed here, allow for a much better representation of the overall process than conventional (purely macroscopic) compartment models. In turn, this enables a better understanding of the process and, in particular, models and model assumptions can be tested in much more detail. Moreover, medical interventions often involve the microstructure, e.g., blocking of receptors, and it is thus expected that such multiscale models will be helpful in this direction as well.

This paper is organized as follows. In section 2, we recall the definitions and some results of the periodic unfolding method and prove the new statements required for the homogenization process, which follows. It is important to note that these results are general in the sense that they could be useful in the homogenization of related problems. In section 3, the system of reaction-diffusion equations is introduced and its relation to carcinogenesis in a human cell is discussed. Further, we show the a priori estimates and the existence of a solution for every $\varepsilon>0$ in section 4 , the technical details of which are relegated to the appendix. The limit for $\varepsilon$ tending to zero is characterized in section 5, where the main result of convergence of solutions of the microscopic model to solutions of the homogenized system is found in Theorem 5.1. We show uniqueness of the limit model in section 6 and give some concluding remarks in section 7 .

2. The periodic unfolding method. Let $\Omega \subset \mathbf{R}^{n}$ be open and bounded and $Y=[0,1)^{n}$ be the unit cell. Further, let $\Omega_{\varepsilon}=\bigcup_{k \in \mathbf{Z}^{n}} \varepsilon(k+Y)$. We recall the definition of the periodic unfolding operator and a compactness result for $H^{1}$ from [9]. Here and in what follows we denote 


$$
\begin{aligned}
L^{2}(\Omega) & =\left\{u: \Omega \rightarrow \mathbf{R} \mid u \text { measurable and } \int_{\Omega} u^{2} \mathrm{~d} x<\infty\right\}, \\
H^{1}(\Omega) & =\left\{\left.u \in L^{2}(\Omega)\left|\int_{\Omega}\right| \nabla u\right|^{2} \mathrm{~d} x<\infty\right\},
\end{aligned}
$$

where $\nabla u$ is the weak derivative; see [12] for this notation.

Let $\Xi_{\varepsilon}:=\left\{\xi \in \mathbf{Z}^{n} \mid \varepsilon(\xi+Y) \subset \Omega\right\}$ and $\hat{\Omega}_{\varepsilon}:=$ interior $\left\{\bigcup_{\xi \in \Xi_{\varepsilon}} \varepsilon(\xi+\bar{Y})\right\}$. For every $z \in \mathbf{R}^{n},[z]_{Y}$ is defined as the unique integer combination $\sum_{i=1}^{n} k_{i} e_{i}$ of the periods such that $\{z\}_{Y}=z-[z]_{Y} \in Y$. The periodic unfolding operator $\mathcal{T}_{\varepsilon}$ is then defined as follows; see [6].

Definition 2.1. Let $\varepsilon>0, \varphi \in L^{p}\left(\Omega_{\varepsilon}\right)$, and $p \in[1, \infty]$. Then, the periodic unfolding operator $\mathcal{T}_{\varepsilon}: L^{p}\left(\Omega_{\varepsilon}\right) \rightarrow L^{p}\left(\mathbf{R}^{n} \times Y\right)$ is defined as

$$
\begin{aligned}
& {\left[\mathcal{T}_{\varepsilon}(\varphi)\right](x, y)=\varphi\left(\varepsilon\left[\frac{x}{\varepsilon}\right]_{Y}+\varepsilon y\right) \text { a.e. for }(x, y) \in \hat{\Omega}_{\varepsilon} \times Y,} \\
& {\left[\mathcal{T}_{\varepsilon}(\varphi)\right](x, y)=0 \text { a.e. for }(x, y) \in \Omega \backslash \hat{\Omega}_{\varepsilon} \times Y .}
\end{aligned}
$$

TheOrem 2.2. For every $\varepsilon>0$, let $\varphi_{\varepsilon}$ be in $H^{1}\left(\Omega_{\varepsilon}\right)$ with $\left\|\varphi_{\varepsilon}\right\|_{H^{1}\left(\Omega_{\varepsilon}\right)}$ bounded independently of $\varepsilon$. Then, there exists $\varphi \in H^{1}(\Omega)$ and $\hat{\varphi} \in L^{2}\left(\Omega, H_{\#}^{1}(Y)\right)$ such that, up to a subsequence,

$$
\begin{aligned}
\mathcal{T}_{\varepsilon}\left(\varphi_{\varepsilon}\right) & \rightarrow \varphi \quad \text { weakly in } L_{\text {loc }}^{2}\left(\Omega, H_{\#}^{1}(Y)\right), \\
\mathcal{T}_{\varepsilon}\left(\nabla_{x} \varphi_{\varepsilon}\right) & -\nabla_{x} \varphi+\nabla_{y} \hat{\varphi} \quad \text { weakly in } L_{\mathrm{loc}}^{2}\left(\Omega, L^{2}(Y)\right) .
\end{aligned}
$$

Functions $\varphi \in L^{2}\left(\Omega, H_{\#}^{1}(Y)\right)$ are $Y$-periodic in their second argument.

Further, let $\Gamma \subset Y$ and $\Gamma_{\varepsilon}=\bigcup_{k \in \mathbf{Z}^{n}} \varepsilon(k+\Gamma)$ be smooth manifolds. The definition of the boundary periodic unfolding operator $\mathcal{T}_{\varepsilon}^{b}$ is given as follows; see [9].

Definition 2.3. Let $\varphi \in L^{p}\left(\Gamma_{\varepsilon}\right), p \in[1, \infty]$. Then, the boundary periodic unfolding operator $\mathcal{T}_{\varepsilon}^{b}: L^{p}\left(\Gamma_{\varepsilon}\right) \rightarrow L^{p}(\Omega \times \Gamma)$ is defined as

$$
\begin{aligned}
& \mathcal{T}_{\varepsilon}^{b}(\varphi)(x, y)=\varphi\left(\varepsilon\left[\frac{x}{\varepsilon}\right]+\varepsilon y\right) \text { a.e. for }(x, y) \in \hat{\Omega}_{\varepsilon} \times \Gamma, \\
& \mathcal{T}_{\varepsilon}^{b}(\varphi)(x, y)=0 \text { a.e. for }(x, y) \in \Omega \backslash \hat{\Omega}_{\varepsilon} \times \Gamma .
\end{aligned}
$$

The boundary periodic unfolding operator has some important properties, summarized in the following lemma, the proofs of which can be found in [9].

Lemma 2.4. For the periodic unfolding operator $\mathcal{T}_{\varepsilon}^{b}$ as defined in Definition 2.3, the following assumptions hold true:

1. $\mathcal{T}_{\varepsilon}^{b}$ is linear.

2. $\mathcal{T}_{\varepsilon}^{b}(\varphi \psi)=\mathcal{T}_{\varepsilon}^{b}(\varphi) \mathcal{T}_{\varepsilon}^{b}(\psi)$ for all $\varphi, \psi \in L^{p}\left(\Gamma_{\varepsilon}\right)$.

3. For every $\varphi \in L^{1}\left(\Gamma_{\varepsilon}\right)$, we have the integration formula

$$
\int_{\Gamma_{\varepsilon}} \varphi(x) \mathrm{d} \sigma_{x}=\frac{1}{\varepsilon|Y|} \int_{\Omega \times \Gamma} \mathcal{T}_{\varepsilon}^{b}(\varphi)(x, y) \mathrm{d} x \mathrm{~d} \sigma_{y} .
$$

The remaining five results of this section for the boundary periodic unfolding operator are new. The first one considers the limit of a product of functions using the periodic unfolding method.

Lemma 2.5. Let $u_{\varepsilon}, v_{\varepsilon} \in L^{2}\left(\Gamma_{\varepsilon}\right)$. Let $\mathcal{T}_{\varepsilon}^{b}\left(u_{\varepsilon}\right)$ converge to $u_{0}$ weakly in $L^{2}(\Omega \times \Gamma)$ and let $\mathcal{T}_{\varepsilon}^{b}\left(v_{\varepsilon}\right)$ converge to $v_{0}$ strongly in $L^{2}(\Omega \times \Gamma)$. Then,

$$
\mathcal{T}_{\varepsilon}^{b}\left(u_{\varepsilon}\right) \mathcal{T}_{\varepsilon}^{b}\left(v_{\varepsilon}\right) \rightarrow u_{0} v_{0}
$$

weakly in $L^{2}(\Omega \times \Gamma)$.

Copyright $@$ by SIAM. Unauthorized reproduction of this article is prohibited. 
Proof. We have for test functions $\varphi \in C^{\infty}(\Omega \times \Gamma)$ that

$$
\begin{aligned}
& \lim _{\varepsilon \rightarrow 0} \int_{\Omega \times \Gamma}\left(\mathcal{T}_{\varepsilon}^{b}\left(u_{\varepsilon}\right) \mathcal{T}_{\varepsilon}^{b}\left(v_{\varepsilon}\right)-u_{0} v_{0}\right) \varphi \mathrm{d} \sigma_{y} \mathrm{~d} x \\
& \quad=\lim _{\varepsilon \rightarrow 0} \int_{\Omega \times \Gamma}\left(\mathcal{T}_{\varepsilon}^{b}\left(u_{\varepsilon}\right) \mathcal{T}_{\varepsilon}^{b}\left(v_{\varepsilon}\right)-\mathcal{T}_{\varepsilon}^{b}\left(u_{\varepsilon}\right) v_{0}+\mathcal{T}_{\varepsilon}^{b}\left(u_{\varepsilon}\right) v_{0}-u_{0} v_{0}\right) \varphi \mathrm{d} \sigma_{y} \mathrm{~d} x \\
& \quad=\lim _{\varepsilon \rightarrow 0} \int_{\Omega \times \Gamma} \mathcal{T}_{\varepsilon}^{b}\left(u_{\varepsilon}\right)\left(\mathcal{T}_{\varepsilon}^{b}\left(v_{\varepsilon}\right)-v_{0}\right) \varphi \mathrm{d} \sigma_{y} \mathrm{~d} x+\lim _{\varepsilon \rightarrow 0} \int_{\Omega \times \Gamma}\left(\mathcal{T}_{\varepsilon}^{b}\left(u_{\varepsilon}\right)-u_{0}\right) \underbrace{v_{0} \varphi}_{\tilde{\varphi}} \mathrm{d} \sigma_{y} \mathrm{~d} x \\
& \quad=\lim _{\varepsilon \rightarrow 0} \int_{\Omega \times \Gamma} \mathcal{T}_{\varepsilon}^{b}\left(u_{\varepsilon}\right)\left(\mathcal{T}_{\varepsilon}^{b}\left(v_{\varepsilon}\right)-v_{0}\right) \varphi \mathrm{d} \sigma_{y} \mathrm{~d} x .
\end{aligned}
$$

Here, we used that $\tilde{\varphi}:=v_{0} \varphi \in L^{2}(\Omega \times \Gamma)$ can be used as test function as well. We continue with the absolute values of the limits:

$$
\begin{aligned}
& \lim _{\varepsilon \rightarrow 0}\left|\int_{\Omega \times \Gamma}\left(\mathcal{T}_{\varepsilon}^{b}\left(u_{\varepsilon}\right) \mathcal{T}_{\varepsilon}^{b}\left(v_{\varepsilon}\right)-u_{0} v_{0}\right) \varphi \mathrm{d} \sigma_{y} \mathrm{~d} x\right| \\
& \quad=\lim _{\varepsilon \rightarrow 0}\left|\int_{\Omega \times \Gamma} \mathcal{T}_{\varepsilon}^{b}\left(u_{\varepsilon}\right)\left(\mathcal{T}_{\varepsilon}^{b}\left(v_{\varepsilon}\right)-v_{0}\right) \varphi \mathrm{d} \sigma_{y} \mathrm{~d} x\right| \\
& \quad \leq \lim _{\varepsilon \rightarrow 0} \underbrace{\left\|\mathcal{T}_{\varepsilon}^{b}\left(u_{\varepsilon}\right) \varphi\right\|_{L^{2}(\Omega \times \Gamma)}}_{\text {bounded }} \underbrace{\left\|\mathcal{T}_{\varepsilon}^{b}\left(v_{\varepsilon}\right)-v_{0}\right\|_{L^{2}(\Omega \times \Gamma)}}_{\rightarrow 0} \\
& \quad=0
\end{aligned}
$$

for every $\varphi \in C^{\infty}(\Omega \times \Gamma)$ and the assertion holds true.

The most useful new result is Theorem 2.9. It allows us to apply the boundary periodic unfolding operator for diffusion equations defined on smooth manifolds. For linear reaction-diffusion equations defined on manifolds it is also possible to use twoscale convergence for the homogenization process; see [23, 2], and we also refer to [15] for results on fast diffusion on manifolds. But if there are nonlinear reaction terms in the equation, strong convergence of the functions typically is required. This is not straightforward on manifolds but an elegant way is by using the boundary periodic unfolding operator, as done in [11]. For nonlinear reaction-diffusion equations on smooth manifolds, Theorem 2.9 can be used, such as in Lemma 4.3 below.

Before we can formulate and prove Theorem 2.9, we first describe a suitable setting. Let $\Gamma \subset \mathbf{R}^{n}$ be a $k$-dimensional compact $C^{\infty}$-Riemannian manifold with Riemannian metric $g$. This means we have an atlas $\left\{\left(U_{\lambda}, \alpha_{\lambda}\right) \mid \lambda \in \Lambda\right\}$ of charts on $\Gamma$ such that $\Gamma=\bigcup_{\lambda} U_{\lambda}$ and

$$
\alpha_{\lambda}: U_{\lambda} \rightarrow V_{\lambda} \subset \mathbf{R}^{k}, \quad \lambda \in \Lambda .
$$

Further, we require that $\Gamma_{\varepsilon}=\bigcup_{\xi \in \mathbf{Z}^{n}} \varepsilon(\Gamma+\xi)$ also is a Riemannian manifold with atlas $\left\{\left(U_{\lambda, \xi}^{\varepsilon}, \alpha_{\lambda, \xi}^{\varepsilon}\right) \mid \lambda \in \Lambda, \xi \in \mathbf{Z}^{n}\right\}$, where $U_{\lambda, \xi}^{\varepsilon}:=\varepsilon\left(U_{\lambda}+\xi\right)$. This means $\Gamma_{\varepsilon}=\bigcup_{\lambda, \xi} U_{\lambda, \xi}^{\varepsilon}$ and

$$
\begin{gathered}
\alpha_{\lambda, \xi}^{\varepsilon}: U_{\lambda, \xi}^{\varepsilon} \rightarrow V_{\lambda}, \\
\alpha_{\lambda, \xi}^{\varepsilon}(p):=\alpha_{\lambda}\left(\frac{1}{\varepsilon} p-\xi\right), \quad p \in U_{\lambda, \xi}^{\varepsilon}, \quad \forall \lambda \in \Lambda, \xi \in \mathbf{Z}^{n}, \varepsilon>0 .
\end{gathered}
$$

Copyright (C) by SIAM. Unauthorized reproduction of this article is prohibited. 
Obviously we have

$$
\alpha_{\lambda, \xi}^{\varepsilon}(p)=\alpha_{\lambda}\left(\left\{\frac{p}{\varepsilon}\right\}_{Y}\right)=\alpha_{\lambda}\left(y_{p}\right), \quad p \in U_{\lambda, \xi}^{\varepsilon}, \quad \forall \lambda \in \Lambda, \xi \in \mathbf{Z}^{n},
$$

where $y_{p}:=\left\{\frac{p}{\varepsilon}\right\}_{Y}$. For the inverse of $\alpha_{\lambda}$ we have $\alpha_{\lambda}^{-1}: V_{\lambda} \rightarrow U_{\lambda}$ and $\alpha_{\lambda, \xi}^{-1, \varepsilon}: V_{\lambda} \rightarrow$ $U_{\lambda, \xi}^{\varepsilon}$ given by

$$
\alpha_{\lambda, \xi}^{-1, \varepsilon}(z)=\varepsilon\left(\alpha_{\lambda}^{-1}(z)+\xi\right)=\pi_{\xi}^{\varepsilon}\left(\alpha_{\lambda}^{-1}(z)\right)
$$

with the function $\pi_{\xi}$ defined as

$$
\pi_{\xi}^{\varepsilon}: \Gamma \rightarrow \Gamma_{\varepsilon}, \quad \pi_{\xi}^{\varepsilon}(y):=\varepsilon(y+\xi), \quad \xi \in \mathbf{Z}^{n} .
$$

For any function $\varphi \in L^{p}\left(\Gamma_{\varepsilon}\right)$ the relation between $\pi_{\xi}$ and $\mathcal{T}_{\varepsilon}^{b}$ is given by

$$
\varphi\left(\pi_{\xi}^{\varepsilon}(y)\right)=\mathcal{T}_{\varepsilon}^{b}(\varphi)(\xi, y)
$$

Now, let us have a look at the tangential vectors $\frac{\mathrm{d}}{\mathrm{d} x^{i, \varepsilon}}$ on $\Gamma_{\varepsilon}$. Let $e_{i}$ be the $i$ th basis vector in $\mathbf{R}^{k}, z=\alpha_{\lambda, \xi}^{\varepsilon}(p) \in V_{\lambda}$ and $t \in[-\delta, \delta], \delta>0$ small. Then, $z=\alpha_{\lambda}\left(y_{p}\right)$ and

$$
t \mapsto z+t e_{i}
$$

is a curve in $V_{\lambda}$. The relationship between tangential vectors on $\Gamma_{\varepsilon}$ and on $\Gamma$ in the point $p$ is given by

$$
\frac{\mathrm{d}}{\mathrm{d} x^{i, \varepsilon}}(p):=\left.\frac{\mathrm{d}}{\mathrm{d} t}\right|_{t=0} \alpha_{\lambda, \xi}^{-1, \varepsilon}\left(z+t e_{i}\right)=\left.\frac{\mathrm{d}}{\mathrm{d} t}\right|_{t=0} \varepsilon \alpha_{\lambda}^{-1}\left(z+t e_{i}\right)=\varepsilon \frac{\mathrm{d}}{\mathrm{d} x^{i}}\left(y_{p}\right) .
$$

Next, we have a look at the Riemannian metrics $g_{i j}$ and $g_{i j}^{\varepsilon}$. We have

$$
\begin{gathered}
g_{i j}^{\varepsilon}(p)=\left\langle\frac{\mathrm{d}}{\mathrm{d} x^{i, \varepsilon}}(p), \frac{\mathrm{d}}{\mathrm{d} x^{j, \varepsilon}}(p)\right\rangle=\left\langle\varepsilon \frac{\mathrm{d}}{\mathrm{d} x^{i}}\left(y_{p}\right), \varepsilon \frac{\mathrm{d}}{\mathrm{d} x^{j}}\left(y_{p}\right)\right\rangle=\varepsilon^{2} g_{i j}\left(y_{p}\right), \\
\text { which yields } g^{i j, \varepsilon}(p)=\frac{1}{\varepsilon^{2}} g^{i j}\left(y_{p}\right)
\end{gathered}
$$

for $i, j=1, \ldots, k$. Within this setting, we want to deduce some assertions. The first one is an extension of the fact that $\nabla_{y} \mathcal{T}_{\varepsilon}\left(\varphi_{\varepsilon}\right)=\varepsilon \mathcal{T}_{\varepsilon}\left(\nabla_{x} \varphi_{\varepsilon}\right)$ for functions $\varphi_{\varepsilon} \in H^{1}\left(\Omega_{\varepsilon}\right)$ (see [9]) to functions on $H^{1}\left(\Gamma_{\varepsilon}\right)$.

Lemma 2.6. Let $\varphi$ be in $H^{1}\left(\Gamma_{\varepsilon}\right)$. Then,

$$
\varepsilon \mathcal{T}_{\varepsilon}^{b}\left(\nabla_{x} \varphi\right)=\nabla_{y} \mathcal{T}_{\varepsilon}^{b}(\varphi)
$$

Proof. In the proof we suppress the $\lambda$ or $\xi$ dependence of the charts $\alpha_{\lambda}$ and $\alpha_{\lambda, \xi}^{\varepsilon}$. We just take the appropriate chart for any subset $U_{\lambda} \subset \Gamma$ and $U_{\lambda, \xi}^{\varepsilon} \subset \Gamma_{\varepsilon}$, respectively. In the setting of Riemannian manifolds the gradient $\nabla_{x} \varphi$ is defined as

$$
\nabla_{x} \varphi(p)=\sum_{i j} g^{i j \varepsilon}(p) \frac{\partial \varphi}{\partial x^{j, \varepsilon}}(p) \frac{\mathrm{d}}{\mathrm{d} x^{i, \varepsilon}}(p)
$$

Copyright $@$ ㅇ by SIAM. Unauthorized reproduction of this article is prohibited. 
with

$$
\frac{\partial \varphi}{\partial x^{i, \varepsilon}}(p):=\frac{\partial\left(\varphi \circ \alpha^{-1, \varepsilon}\right)}{\partial x_{i}}\left(\alpha^{\varepsilon}(p)\right)
$$

Here $x_{i}, i=1, \ldots, k$, denote the components of $\mathbf{R}^{k}$. Applying $\mathcal{T}_{\varepsilon}^{b}$ to $\frac{\partial \varphi}{\partial x^{j, \varepsilon}}$ leads to

$$
\begin{aligned}
\mathcal{T}_{\varepsilon}^{b}\left(\frac{\partial \varphi}{\partial x^{j, \varepsilon}}\right)\left(p, y_{p}\right) & =\left(\frac{\partial\left(\varphi \circ \alpha^{-1, \varepsilon}\right)}{\partial x_{j}} \circ \alpha^{\varepsilon}\right)\left([p]_{Y}+\varepsilon y_{p}\right) \\
& =\frac{\partial\left(\varphi \circ \pi \circ \alpha^{-1}\right)}{\partial x_{j}} \alpha\left(y_{p}\right)=\frac{\partial_{y}\left(\mathcal{T}_{\varepsilon}^{b}(\varphi) \circ \alpha^{-1}\right)}{\partial x_{j}} \alpha\left(y_{p}\right) \\
& =\frac{\partial_{y} \mathcal{T}_{\varepsilon}^{b}(\varphi)}{\partial x^{j}}\left(p, y_{p}\right) .
\end{aligned}
$$

Putting the pieces together we get

$$
\begin{aligned}
\mathcal{T}_{\varepsilon}^{b}\left(\nabla_{x} \varphi\right)\left(p, y_{p}\right) & =\mathcal{T}_{\varepsilon}^{b}\left(\sum_{i j} g^{i j \varepsilon} \frac{\partial \varphi}{\partial x^{j, \varepsilon}} \frac{\mathrm{d}}{\mathrm{d} x^{i, \varepsilon}}\right)\left(p, y_{p}\right) \\
& =\sum_{i j} \frac{1}{\varepsilon^{2}} g^{i j}\left(y_{p}\right) \mathcal{T}_{\varepsilon}^{b}\left(\frac{\partial \varphi}{\partial x^{j, \varepsilon}}\right)\left(p, y_{p}\right) \varepsilon \frac{\mathrm{d}}{\mathrm{d} x^{i}}\left(y_{p}\right)=\frac{1}{\varepsilon} \nabla_{y}\left(\mathcal{T}_{\varepsilon}^{b}(\varphi)\right)\left(p, y_{p}\right) .
\end{aligned}
$$

Thus, $\nabla_{y}\left(\mathcal{T}_{\varepsilon}^{b}(\varphi)\right)=\varepsilon \mathcal{T}_{\varepsilon}^{b}\left(\nabla_{x} \varphi\right) . \quad \square$

Having established this result, the following two lemmas easily follow.

Lemma 2.7. Let $\varphi$ be in $H^{1}\left(\Gamma_{\varepsilon}\right)$. Then,

$$
\left\|\nabla_{y} \mathcal{T}_{\varepsilon}^{b}(\varphi)\right\|_{L^{2}\left(\mathbf{R}^{n} \times \Gamma\right)}^{2}=|Y| \varepsilon^{3}\left\|\nabla_{x} \varphi\right\|_{L^{2}\left(\Gamma_{\varepsilon}\right)}^{2} .
$$

Proof. With assertion 3 of Lemma 2.4 we have

$$
\begin{aligned}
\left\|\nabla_{x} \varphi\right\|_{L^{2}\left(\Gamma_{\varepsilon}\right)}^{2} & =\frac{1}{\varepsilon|Y|} \int_{\mathbf{R}^{n} \times \Gamma} \mathcal{T}_{\varepsilon}^{b}\left(\nabla_{x} \varphi\right) \mathcal{T}_{\varepsilon}^{b}\left(\nabla_{x} \varphi\right) \mathrm{d} x \mathrm{~d} \sigma_{y} \\
& =\frac{1}{\varepsilon|Y|} \int_{\mathbf{R}^{n} \times \Gamma} \frac{1}{\varepsilon} \nabla_{y} \mathcal{T}_{\varepsilon}^{b}(\varphi) \frac{1}{\varepsilon} \nabla_{y} \mathcal{T}_{\varepsilon}^{b}(\varphi) \mathrm{d} x \mathrm{~d} \sigma_{y}=\frac{1}{\varepsilon^{3}|Y|}\left\|\nabla_{y} \mathcal{T}_{\varepsilon}^{b}(\varphi)\right\|_{L^{2}\left(\mathbf{R}^{n} \times \Gamma\right)}^{2}
\end{aligned}
$$

and the claim follows.

Lemma 2.8. If $\varphi_{\varepsilon} \in H^{1}\left(\Gamma_{\varepsilon}\right)$, then $\mathcal{T}_{\varepsilon}^{b}\left(\varphi_{\varepsilon}\right) \in L^{2}\left(\Omega, H^{1}(\Gamma)\right)$.

Proof. Since $\varphi_{\varepsilon} \in H^{1}\left(\Gamma_{\varepsilon}\right)$, it holds that

$$
\varepsilon\left\|\varphi_{\varepsilon}\right\|_{L^{2}\left(\Gamma_{\varepsilon}\right)}^{2}+\varepsilon\left\|\nabla_{x} \varphi_{\varepsilon}\right\|_{L^{2}\left(\Gamma_{\varepsilon}\right)}^{2} \leq C(\varepsilon)
$$

for a $C(\varepsilon)>0$. Because $\nabla_{y} \mathcal{T}_{\varepsilon}^{b}\left(\varphi_{\varepsilon}\right)=\varepsilon \mathcal{T}_{\varepsilon}^{b}\left(\nabla_{x} \varphi_{\varepsilon}\right)$ we have for small $\varepsilon<1$

$$
\begin{aligned}
\left\|\mathcal{T}_{\varepsilon}^{b}\left(\varphi_{\varepsilon}\right)\right\|_{L^{2}\left(\Omega, H^{1}(\Gamma)\right)}^{2} & =\int_{\Omega \times \Gamma} \mathcal{T}_{\varepsilon}^{b}\left(\varphi_{\varepsilon}\right)^{2} \mathrm{~d} \sigma_{y} \mathrm{~d} x+\int_{\Omega \times \Gamma}\left(\nabla_{y} \mathcal{T}_{\varepsilon}^{b}\left(\varphi_{\varepsilon}\right)\right)^{2} \mathrm{~d} \sigma_{y} \mathrm{~d} x \\
& =|Y| \varepsilon\left\|\varphi_{\varepsilon}\right\|_{L^{2}\left(\Gamma_{\varepsilon}\right)}^{2}+|Y| \varepsilon^{3}\left\|\nabla_{x} \varphi_{\varepsilon}\right\|_{L^{2}\left(\Gamma_{\varepsilon}\right)}^{2} \leq|Y| C(\varepsilon) .
\end{aligned}
$$

The main Theorem 2.9 is a compactness result and deduces a limit function in $L^{2}\left(\Omega, H_{\#}^{1}(\Gamma)\right)$ of a bounded sequence in $H^{1}\left(\Gamma_{\varepsilon}\right)$.

TheOREM 2.9. Let $\varphi_{\varepsilon} \in H^{1}\left(\Gamma_{\varepsilon}\right)$ be bounded for every $\varepsilon$ such that

$$
\varepsilon\left\|\varphi_{\varepsilon}\right\|_{L^{2}\left(\Gamma_{\varepsilon}\right)}^{2} \leq C \quad \text { and } \quad \varepsilon^{3}\left\|\nabla_{x} \varphi_{\varepsilon}\right\|_{L^{2}\left(\Gamma_{\varepsilon}\right)}^{2} \leq C
$$

Copyright (c) by SIAM. Unauthorized reproduction of this article is prohibited. 
for $C>0$ independent of $\varepsilon$. Then, there exists a $\hat{\varphi} \in L^{2}\left(\mathbf{R}^{n}, H_{\#}^{1}(\Gamma)\right)$ such that, up to a subsequence,

$$
\mathcal{T}_{\varepsilon}^{b}\left(\varphi_{\varepsilon}\right) \rightarrow \hat{\varphi} \quad \text { weakly in } L^{2}\left(\mathbf{R}^{n}, H^{1}(\Gamma)\right)
$$

and

$$
\varepsilon \mathcal{T}_{\varepsilon}^{b}\left(\nabla_{x} \varphi_{\varepsilon}\right) \rightarrow \nabla_{y} \hat{\varphi} \quad \text { weakly in } L^{2}\left(\mathbf{R}^{n} \times \Gamma\right) .
$$

Proof. We use the statement that, in a reflexive Banach space, a bounded sequence contains a weakly converging subsequence. Hence, we need to show that $\mathcal{T}_{\varepsilon}^{b}\left(\varphi_{\varepsilon}\right)$ is bounded in $L^{2}\left(\mathbf{R}^{n}, H^{1}(\Gamma)\right)$.

With assertion 3 of Lemma 2.4 we get

$$
\frac{1}{|Y|}\left\|\mathcal{T}_{\varepsilon}^{b}\left(\varphi_{\varepsilon}\right)\right\|_{L^{2}\left(\mathbf{R}^{n} \times \Gamma\right)}^{2}=\varepsilon\left\|\varphi_{\varepsilon}\right\|_{L^{2}\left(\Gamma_{\varepsilon}\right)}^{2} \leq C
$$

and with Lemma 2.7

$$
\frac{1}{|Y|}\left\|\nabla_{y} \mathcal{T}_{\varepsilon}^{b}\left(\varphi_{\varepsilon}\right)\right\|_{L^{2}\left(\mathbf{R}^{n} \times \Gamma\right)}^{2}=\varepsilon^{3}\left\|\nabla_{x} \varphi_{\varepsilon}\right\|_{L^{2}\left(\Gamma_{\varepsilon}\right)}^{2} \leq C .
$$

Hence, $\mathcal{T}_{\varepsilon}^{b}\left(\varphi_{\varepsilon}\right)$ is bounded in $L^{2}\left(\mathbf{R}^{n} \times \Gamma\right)$ and $\nabla_{y} \mathcal{T}_{\varepsilon}^{b}(\varphi)$ is bounded in $L^{2}\left(\mathbf{R}^{n} \times \Gamma\right)$. It follows that $\mathcal{T}_{\varepsilon}^{b}\left(\varphi_{\varepsilon}\right)$ is bounded in $L^{2}\left(\mathbf{R}^{n}, H^{1}(\Gamma)\right)$ and there exists $\hat{\varphi} \in L^{2}\left(\mathbf{R}^{n}, H^{1}(\Gamma)\right)$ such that

$$
\mathcal{T}_{\varepsilon}^{b}\left(\varphi_{\varepsilon}\right) \rightarrow \hat{\varphi} \quad \text { weakly in } L^{2}\left(\mathbf{R}^{n}, H^{1}(\Gamma)\right) .
$$

With Lemma 2.6 we conclude

$$
\varepsilon \mathcal{T}_{\varepsilon}^{b}\left(\nabla_{x} \varphi_{\varepsilon}\right)=\nabla_{y} \mathcal{T}_{\varepsilon}^{b}\left(\varphi_{\varepsilon}\right) \rightarrow \nabla_{y} \hat{\varphi} \quad \text { weakly in } L^{2}\left(\mathbf{R}^{n} \times \Gamma\right) .
$$

It is left to show that $\hat{\varphi}$ is $Y$-periodic. To this end, let $\psi \in C^{\infty}\left(\mathbf{R}^{n} \times \Gamma\right)$ be periodic in its second argument. Then, for $\xi \in \mathbf{Z}^{n}$

$$
\begin{aligned}
\int_{\mathbf{R}^{n} \times \Gamma}\left(\mathcal{T}_{\varepsilon}^{b}\left(\varphi_{\varepsilon}\right)(x, y+\xi)-\mathcal{T}_{\varepsilon}^{b}\left(\varphi_{\varepsilon}\right)(x, y)\right) \psi(x, y) \mathrm{d} x \mathrm{~d} \sigma_{y} \\
=\int_{\mathbf{R}^{n} \times \Gamma}\left(\varphi_{\varepsilon}\left(\varepsilon\left(\left[\frac{x}{\varepsilon}\right]_{Y}+\xi\right)+\varepsilon y\right)-\varphi_{\varepsilon}\left(\varepsilon\left[\frac{x}{\varepsilon}\right]_{Y}+\varepsilon y\right)\right) \psi(x, y) \mathrm{d} x \mathrm{~d} \sigma_{y} \\
=\int_{\mathbf{R}^{n} \times \Gamma} \varphi_{\varepsilon}\left(\varepsilon\left[\frac{x}{\varepsilon}\right]_{Y}+\varepsilon y\right)(\psi(x-\varepsilon \xi, y)-\psi(x, y)) \mathrm{d} x \mathrm{~d} \sigma_{y} .
\end{aligned}
$$

Since $\psi(x-\varepsilon \xi, y) \rightarrow \psi(x, y)$ for $\varepsilon$ tending to zero, we finally conclude that

$$
\int_{\mathbf{R}^{n} \times \Gamma} \mathcal{T}_{\varepsilon}^{b}\left(\varphi_{\varepsilon}\right)(x, y+\xi) \psi(x, y) \mathrm{d} x \mathrm{~d} \sigma_{y}-\int_{\mathbf{R}^{n} \times \Gamma} \mathcal{T}_{\varepsilon}^{b}\left(\varphi_{\varepsilon}\right)(x, y) \psi(x, y) \mathrm{d} x \mathrm{~d} \sigma_{y} \stackrel{\varepsilon \rightarrow 0}{\longrightarrow} 0 .
$$

3. Nonlinear carcinogenesis problem. With the tools introduced in the previous section, we are prepared to pass to the homogenization limit of a system of equations including diffusion on a biological membrane using the periodic unfolding method. One of the longest known and best understood causes of carcinogenesis is the molecule Benzo[a]pyrene (BP). It is found, for example, in coal tar, automobile exhaust fumes, cigarette smoke, and charbroiled food. One of the main reasons for 
lung cancer (caused by inhaling cigarette smoke), testicular cancer, and skin cancer is the contact with the molecule BP. Often chimney sweepers are affected because of the frequent exposure to coal (see $[20,14]$ ).

The molecule itself is not dangerous. But chemical reactions in the human cell can transform it to the molecule Benzo[a]pyrene-7,8-diol-9,10-epoxide (DE), which can bind to and damage the human DNA (see [14]). The chemical reactions mostly take place on the surface of the endoplasmic reticulum.

In the cytosol of a human cell, there are molecules which can bind to $\mathrm{BP}$ or $\mathrm{DE}$ and render them harmless. Examples of such molecules are glutathione epoxide transferase or sulfo transferase (transferase is an enzyme); see [13]. They bind to potentially dangerous and alien molecules and render them water soluble.

Hence, the process of toxification is simplified by the following scenario. BP molecules pass the plasma membrane from the intercellular space to the cytosol inside of a human cell, where they diffuse freely and can be removed by cleaning mechanisms of the cell. They can bind to the surface of the endoplasmic reticulum by connecting to receptors. There, a series of chemical reactions takes place summarized to just one metabolism from BP to DE. Newly created DE molecules unbind from the surface of the endoplasmic reticulum by uncoupling from the receptor and diffuse again in the cytosol of the cell, where they can be removed by cleaning mechanisms. There, they may enter the nucleus. For simplicity, we restrict BP not to pass the nuclear membrane, whereas DE cannot pass the plasma membrane, which describes a worstcase scenario.

3.1. Microscopic model. Let $\Omega \subset \mathbf{R}^{n}$ be a human cell with a Lipschitz boundary $\partial \Omega$ and which we assume to be representable by a finite union of axis-parallel cuboids with corner coordinates in $\mathbb{Q}^{n}$. Furthermore, let $Y=[0,1)^{n}$ be a unit cell with an open subset $Y_{0} \subset Y$ with smooth boundary $\Gamma$, where $\Gamma$ does not touch the boundary of $Y$. The sets $Y^{*}=Y \backslash \overline{Y_{0}}$ and $\Gamma$ form characteristic parts of the cytosol and the surface of the endoplasmic reticulum, respectively. Let $\varepsilon>0$; then $\Omega_{\varepsilon}:=\bigcup_{k \in \mathbf{Z}^{n}} \varepsilon\left(k+Y^{*}\right) \cap \Omega$ is $\varepsilon$-periodic and $\Gamma_{\varepsilon}:=\bigcup_{k \in \mathbf{Z}^{n}} \varepsilon(k+\Gamma) \cap \Omega$ a periodic and smooth surface. The process is considered in the time interval $[0, T]$ for fixed $0<T<\infty$. Further, the concentration of BP molecules in cytosol is denoted by $u_{\varepsilon}:[0, T] \times \Omega_{\varepsilon} \rightarrow \mathbf{R}$ and the concentration of DE molecules in cytosol is $v_{\varepsilon}:[0, T] \times \Omega_{\varepsilon} \rightarrow \mathbf{R}$. The concentration of BP molecules bound to the surface of the endoplasmic reticulum is denoted by $s_{\varepsilon}:[0, T] \times \Gamma_{\varepsilon} \rightarrow \mathbf{R}$ and the concentration of DE molecules bound to the surface of the endoplasmic reticulum is denoted by $w_{\varepsilon}:[0, T] \times \Gamma_{\varepsilon} \rightarrow \mathbf{R}$. The relative concentration of free receptors on the surface of the endoplasmic reticulum is given by $R_{\varepsilon}:[0, T] \times \Gamma_{\varepsilon} \rightarrow[0,1]$. Molecules bind to a membrane by connecting to receptors, which are attached to the membrane. BP molecules in the cytosol $\left(u_{\varepsilon}\right)$ can transform to BP molecules bound to the surface of the ER $\left(s_{\varepsilon}\right)$ only when they find a free receptor $\left(R_{\varepsilon}\right)$. The maximal relative amount of free receptors is denoted by $\bar{R}=1$.

This consideration leads to the following microscopic model for carcinogenesis of a human cell in the context described above. BP molecules diffuse freely in the cytosol with diffusion coefficient $D_{u}>0$ and the cleaning mechanism is taken care of by the function $f$,

$$
\partial_{t} u_{\varepsilon}-D_{u} \Delta u_{\varepsilon}=-f\left(u_{\varepsilon}\right) \text { in } \Omega_{\varepsilon} .
$$

The enzymes necessary for cleaning are available only in limited quantities. If only a few $\mathrm{BP}$ molecules are present, we assume that the cleaning is almost linear. If there 
are many molecules the cleaning rate will reach a threshold. The following function is suitable to describe this behavior:

$$
f: \mathbf{R} \rightarrow \mathbf{R}_{0}^{+}, \quad f(x)=\left\{\begin{array}{cl}
\frac{x}{x+M} M a & \text { for } x \geq 0, \\
0 & \text { for } x<0,
\end{array}\right.
$$

where $M, a>0$. This function $f$ is nonlinear, nonnegative, bounded, and Lipschitzcontinuous. At the surface of the endoplasmic reticulum, BP molecules can bind to receptors. With the law of mass action, the binding is modeled by the product $k_{u} u_{\varepsilon} R_{\varepsilon}$, since one BP molecule and one receptor are needed, with constant rate $k_{u}>0$. Bound $\mathrm{BP}$ molecules are denoted by $s_{\varepsilon}$ and unbind with rate $l_{s}>0$. This Robin-boundary term is multiplied by $\varepsilon$ to compensate the growth of the surface by shrinking $\varepsilon$ (see [23] for details),

$$
-D_{u} \nabla u_{\varepsilon} \cdot n=\varepsilon\left(k_{u} R_{\varepsilon} u_{\varepsilon}-l_{s} s_{\varepsilon}\right) \quad \text { on } \Gamma_{\varepsilon} .
$$

At the plasma membrane, $u_{\varepsilon}$ satisfies a Dirichlet boundary condition and at the nuclear membrane a no-flux Neumann boundary condition,

$$
\begin{aligned}
u_{\varepsilon} & =u_{\text {Boundary }} & & \text { on } \Gamma_{\mathrm{C}}, \\
-D_{u} \nabla u_{\varepsilon} \cdot n & =0 & & \text { on } \Gamma_{\mathrm{N}} .
\end{aligned}
$$

DE molecules have a similar behavior, diffuse freely in the cytosol with diffusion coefficient $D_{v}>0$ and cleaning function $g$, which has the same form as $f$ but with different parameters. At the plasma membrane DE molecules satisfy a no-flux Neumann boundary condition and at the nuclear membrane a Dirichlet boundary condition,

$$
\begin{aligned}
\partial_{t} v_{\varepsilon}-D_{v} \Delta v_{\varepsilon} & =-g\left(v_{\varepsilon}\right) & & \text { in } \Omega_{\varepsilon}, \\
-D_{v} \nabla v_{\varepsilon} \cdot n & =\varepsilon\left(k_{v} R_{\varepsilon} v_{\varepsilon}-l_{w} w_{\varepsilon}\right) & & \text { on } \Gamma_{\varepsilon}, \\
-D_{v} \nabla v_{\varepsilon} \cdot n & =0 & & \text { on } \Gamma_{\mathrm{C}}, \\
v_{\varepsilon} & =0 & & \text { on } \Gamma_{\mathrm{N}},
\end{aligned}
$$

with binding and unbinding rates $k_{v}$ and $l_{w}$, respectively. Bound to the endoplasmic reticulum, the molecules diffuse on the surface modeled by the Laplace-Beltrami operator $\Delta_{\Gamma}$. For the transformation from BP molecules to DE molecules bound to the surface of the endoplasmic reticulum, a function $h$ of the same form as $f$ or $g$ is used, since the enzymes necessary for the transformation are available only in limited quantities,

$$
\begin{aligned}
\partial_{t} s_{\varepsilon}-\varepsilon^{2} D_{s} \Delta_{\Gamma} s_{\varepsilon} & =-h\left(s_{\varepsilon}\right)+k_{u} R_{\varepsilon} u_{\varepsilon}-l_{s} s_{\varepsilon} & & \text { on } \Gamma_{\varepsilon}, \\
\partial_{t} w_{\varepsilon}-\varepsilon^{2} D_{w} \Delta_{\Gamma} w_{\varepsilon} & =h\left(s_{\varepsilon}\right)+k_{v} R_{\varepsilon} v_{\varepsilon}-l_{w} w_{\varepsilon} & & \text { on } \Gamma_{\varepsilon} .
\end{aligned}
$$

If BP molecules $u_{\varepsilon}$ or DE molecules $v_{\varepsilon}$ bind to the surface of the endoplasmic reticulum with rate $k_{u}$ or $k_{v}$, the number of free receptors $R_{\varepsilon}$ decreases. If $\mathrm{BP}$ molecules $s_{\varepsilon}$ or DE molecules $w_{\varepsilon}$ leave the surface of the endoplasmic reticulum with rate $l_{s}$ or $l_{w}$, then $R_{\varepsilon}$ increases. Receptors do not move on the surface of the endoplasmic reticulum but are fixed. Hence the equation for $R_{\varepsilon}$ is given by

$$
\partial_{t} R_{\varepsilon}=-R_{\varepsilon}\left|k_{u} u_{\varepsilon}+k_{v} v_{\varepsilon}\right|+\left(\bar{R}-R_{\varepsilon}\right)\left|k_{s} s_{\varepsilon}+k_{w} w_{\varepsilon}\right| \quad \text { on } \Gamma_{\varepsilon} .
$$

The factors $k_{s}>0$ and $k_{w}>0$ are multiples of $l_{s}, l_{w}$, respectively, and ensure that $k_{s}\left(\bar{R}-R_{\varepsilon}\right)$ and $k_{w}\left(\bar{R}-R_{\varepsilon}\right)$ are rates. 
Here, we remark on a simplifying assumption of this formulation of the model. On the surface of the membrane, the molecules diffuse by moving from one free receptor to the next free one. This means that for parts of the membrane, which are crowded with molecules, diffusion of these molecules becomes more difficult because of the lack of free receptors. We neglect this aggregation effect in this model.

The initial values

$$
\left(u_{I}, v_{I}, s_{I}, w_{I}, R_{I}\right)=\left(u_{\varepsilon}(0), v_{\varepsilon}(0), s_{\varepsilon}(0), w_{\varepsilon}(0), \bar{R}\right)
$$

are smooth, bounded, and nonnegative.

For the weak formulation, we take the function spaces

$$
\begin{aligned}
\mathcal{V}_{\mathrm{N}}\left(\Omega_{\varepsilon}\right) & =\left\{u \in L^{2}\left([0, T], H^{1}\left(\Omega_{\varepsilon}\right)\right) \mid u=0 \text { on } \Gamma_{\mathrm{N}}, \partial_{t} u \in L^{2}\left([0, T], H^{1}\left(\Omega_{\varepsilon}\right)^{\prime}\right)\right\}, \\
\mathcal{V}_{\mathrm{C}}\left(\Omega_{\varepsilon}\right) & =\left\{u \in L^{2}\left([0, T], H^{1}\left(\Omega_{\varepsilon}\right)\right) \mid u=u_{\text {Boundary }} \text { on } \Gamma_{\mathrm{C}}, \partial_{t} u \in L^{2}\left([0, T], H^{1}\left(\Omega_{\varepsilon}\right)^{\prime}\right)\right\}, \\
\mathcal{V}\left(\Gamma_{\varepsilon}\right) & =\left\{u \in L^{2}\left([0, T], H^{1}\left(\Gamma_{\varepsilon}\right)\right) \mid \partial_{t} u \in L^{2}\left([0, T], H^{1}\left(\Gamma_{\varepsilon}\right)^{\prime}\right)\right\}, \\
\mathcal{V}_{R}\left(\Gamma_{\varepsilon}\right) & =\left\{u \in L^{2}\left([0, T], L^{2}\left(\Gamma_{\varepsilon}\right)\right) \mid \partial_{t} u \in L^{2}\left([0, T], L^{2}\left(\Gamma_{\varepsilon}\right)\right)\right\}
\end{aligned}
$$

and we use the notation $(\varphi, \psi)_{\Omega_{\varepsilon}}=\int_{\Omega_{\varepsilon}} \varphi \psi \mathrm{d} x,(\varphi, \psi)_{\Omega_{\varepsilon}, t}=\int_{0}^{t}(\varphi(\tau), \psi(\tau))_{\Omega_{\varepsilon}} \mathrm{d} \tau$, and $\langle\varphi, \psi\rangle_{\Gamma_{\varepsilon}}=\int_{\Gamma_{\varepsilon}} g_{\varepsilon} \varphi \psi \mathrm{d} \sigma_{x}$ with Riemannian metric $g_{\varepsilon}$. For the test functions, we need the spaces

$$
\begin{aligned}
V_{\mathrm{C} 0}\left(\Omega_{\varepsilon}\right) & =\left\{u \in H^{1}\left(\Omega_{\varepsilon}\right) \mid u=0 \text { on } \Gamma_{C}\right\}, \\
V_{\mathrm{N}}\left(\Omega_{\varepsilon}\right) & =\left\{u \in H^{1}\left(\Omega_{\varepsilon}\right) \mid u=0 \text { on } \Gamma_{N}\right\}, \\
V\left(\Gamma_{\varepsilon}\right) & =H^{1}\left(\Gamma_{\varepsilon}\right) .
\end{aligned}
$$

Then, the weak formulation is as follows: Find $u_{\varepsilon} \in \mathcal{V}_{\mathrm{C}}\left(\Omega_{\varepsilon}\right), v_{\varepsilon} \in \mathcal{V}_{\mathrm{N}}\left(\Omega_{\varepsilon}\right), s_{\varepsilon}, w_{\varepsilon} \in$ $\mathcal{V}\left(\Gamma_{\varepsilon}\right)$, and $R_{\varepsilon} \in \mathcal{V}_{R}\left(\Gamma_{\varepsilon}\right)$ satisfying the initial condition (3.2a) and

$$
\begin{aligned}
& \left(\partial_{t} u_{\varepsilon}, \varphi_{1}\right)_{\Omega_{\varepsilon}}+D_{u}\left(\nabla u_{\varepsilon}, \nabla \varphi_{1}\right)_{\Omega_{\varepsilon}}+\varepsilon\left\langle k_{u} u_{\varepsilon} R_{\varepsilon}-l_{s} s_{\varepsilon}, \varphi_{1}\right\rangle_{\Gamma_{\varepsilon}}=-\left(f\left(u_{\varepsilon}\right), \varphi_{1}\right)_{\Omega_{\varepsilon}}, \\
& \left(\partial_{t} v_{\varepsilon}, \varphi_{2}\right)_{\Omega_{\varepsilon}}+D_{v}\left(\nabla v_{\varepsilon}, \nabla \varphi_{2}\right)_{\Omega_{\varepsilon}}+\varepsilon\left\langle k_{v} v_{\varepsilon} R_{\varepsilon}-l_{w} w_{\varepsilon}, \varphi_{2}\right\rangle_{\Gamma_{\varepsilon}}=-\left(g\left(v_{\varepsilon}\right), \varphi_{2}\right)_{\Omega_{\varepsilon}}, \\
& \left\langle\partial_{t} s_{\varepsilon}, \psi\right\rangle_{\Gamma_{\varepsilon}}+\varepsilon^{2} D_{s}\left\langle\nabla_{\Gamma} s_{\varepsilon}, \nabla_{\Gamma} \psi\right\rangle_{\Gamma_{\varepsilon}}=\left\langle k_{u} u_{\varepsilon} R_{\varepsilon}-l_{s} s_{\varepsilon}, \psi\right\rangle_{\Gamma_{\varepsilon}}-\left\langle h\left(s_{\varepsilon}\right), \psi\right\rangle_{\Gamma_{\varepsilon}}, \\
& \left\langle\partial_{t} w_{\varepsilon}, \psi\right\rangle_{\Gamma_{\varepsilon}}+\varepsilon^{2} D_{w}\left\langle\nabla_{\Gamma} w_{\varepsilon}, \nabla_{\Gamma} \psi\right\rangle_{\Gamma_{\varepsilon}}=\left\langle k_{v} v_{\varepsilon} R_{\varepsilon}-l_{w} w_{\varepsilon}, \psi\right\rangle_{\Gamma_{\varepsilon}}+\left\langle h\left(s_{\varepsilon}\right), \psi\right\rangle_{\Gamma_{\varepsilon}}, \\
& \left\langle\partial_{t} R_{\varepsilon}, \psi\right\rangle_{\Gamma_{\varepsilon}}+\left\langle R_{\varepsilon}\left|k_{u} u_{\varepsilon}+k_{v} v_{\varepsilon}\right|, \psi\right\rangle_{\Gamma_{\varepsilon}}=\left\langle\left(\bar{R}-R_{\varepsilon}\right)\left|k_{s} s_{\varepsilon}+k_{w} w_{\varepsilon}\right|, \psi\right\rangle_{\Gamma_{\varepsilon}}
\end{aligned}
$$

for all $\left(\varphi_{1}, \varphi_{2}, \psi\right) \in V_{\mathrm{C} 0}\left(\Omega_{\varepsilon}\right) \times V_{\mathrm{N}}\left(\Omega_{\varepsilon}\right) \times V\left(\Gamma_{\varepsilon}\right)$.

We show in what follows that the solution of (3.2) converges to the solution of the homogenized macroscopic system (5.5) in the limit as $\varepsilon \rightarrow 0$, cf. Theorem 5.1.

4. A priori estimates and existence of solutions of the microscopic problem. In this section, we show that the functions $u_{\varepsilon}, v_{\varepsilon}, s_{\varepsilon}, w_{\varepsilon}$, and $R_{\varepsilon}$ are bounded independently of $\varepsilon$ in $L^{2}\left([0, T], H^{1}\left(\Omega_{\varepsilon}\right)\right)$ and $L^{2}\left([0, T], H^{1}\left(\Gamma_{\varepsilon}\right)\right)$ and $L^{2}\left([0, T] \times \Gamma_{\varepsilon}\right)$, respectively. This is necessary to use the periodic unfolding operator for the convergence. Furthermore, we prove that $u_{\varepsilon}$ and $v_{\varepsilon}$ are elements of $L^{\infty}\left([0, T] \times \Omega_{\varepsilon}\right) \cap$ $H^{1}\left([0, T], H_{0}^{1}\left(\Omega_{\varepsilon}\right)^{\prime}\right)$ bounded independently of $\varepsilon$ and that $\mathcal{T}_{\varepsilon}^{b}\left(s_{\varepsilon}\right), \mathcal{T}_{\varepsilon}^{b}\left(w_{\varepsilon}\right)$ and $\mathcal{T}_{\varepsilon}^{b}\left(R_{\varepsilon}\right)$ are Cauchy-sequences in $L^{2}([0, T] \times \Omega \times \Gamma)$. This yields strong convergence of the function sequences. Finally, existence of solutions of the microscopic problem is established.

LEMma 4.1. A function $R_{\varepsilon}$, satisfying (3.2), is nonnegative and bounded by $\bar{R}>0$ almost everywhere in $x \in \Gamma_{\varepsilon}$ and $t \in[0, T]$. 
Proof. First the nonnegativity of $R_{\varepsilon}$ is proved: The weak formulation of $R_{\varepsilon}$ is tested with the function $R_{\varepsilon-}=-R_{\varepsilon}$ for $R_{\varepsilon} \leq 0$ and 0 otherwise, and it is found that $\left\langle\partial_{t} R_{\varepsilon-}, R_{\varepsilon-}\right\rangle_{\Gamma_{\varepsilon}}+\left\langle R_{\varepsilon-}\left|k_{u} u_{\varepsilon}+k_{v} v_{\varepsilon}\right|, R_{\varepsilon-}\right\rangle_{\Gamma_{\varepsilon}}+\left\langle\left(\bar{R}+R_{\varepsilon-}\right)\left|k_{s} s_{\varepsilon}+k_{w} w_{\varepsilon}\right|, R_{\varepsilon-}\right\rangle_{\Gamma_{\varepsilon}}=0$.

Integration from 0 to $t$ and observing $R_{\varepsilon}(0) \geq 0$ yields

$$
\frac{1}{2}\left\|R_{\varepsilon-}\right\|_{\Gamma_{\varepsilon}}^{2}+\underbrace{\left\|R_{\varepsilon-\sqrt{\left|k_{u} u_{\varepsilon}+k_{v} v_{\varepsilon}\right|}}\right\|_{\Gamma_{\varepsilon}, t}^{2}}_{\geq 0}+\underbrace{\left\langle\left(\bar{R}+R_{\varepsilon-}\right)\left|k_{s} s_{\varepsilon}+k_{w} w_{\varepsilon}\right|, R_{\varepsilon-}\right\rangle_{\Gamma_{\varepsilon}, t}}_{\geq 0}=0 .
$$

We deduce $\left\|R_{\varepsilon-}\right\|_{\Gamma_{\varepsilon}}^{2} \leq 0$ for almost every $t \in[0, T]$. This implies $R_{\varepsilon} \geq 0$ for almost every $x \in \Gamma_{\varepsilon}$ and $t \in[0, T]$. To show boundedness of $R_{\varepsilon}$, we test the weak formulation with $\left(R_{\varepsilon}-\bar{R}\right)_{+}=R_{\varepsilon}-\bar{R}$ for $R_{\varepsilon}-\bar{R} \geq 0$ and 0 otherwise and obtain

$$
\begin{gathered}
\left\langle\partial_{t} R_{\varepsilon},\left(R_{\varepsilon}-\bar{R}\right)_{+}\right\rangle_{\Gamma_{\varepsilon}}+\underbrace{\left\langle R_{\varepsilon}\left|k_{u} u_{\varepsilon}+k_{v} v_{\varepsilon}\right|,\left(R_{\varepsilon}-\bar{R}\right)_{+}\right\rangle_{\Gamma_{\varepsilon}}}_{\geq 0} \\
-\left\langle\left(\bar{R}-R_{\varepsilon}\right)\left|k_{s} s_{\varepsilon}+k_{w} w_{\varepsilon}\right|,\left(R_{\varepsilon}-\bar{R}\right)_{+}\right\rangle_{\Gamma_{\varepsilon}}=0 .
\end{gathered}
$$

Since $\partial_{t} \bar{R}=0$, it yields

$$
\left\langle\partial_{t}\left(R_{\varepsilon}-\bar{R}\right)_{+},\left(R_{\varepsilon}-\bar{R}\right)_{+}\right\rangle_{\Gamma_{\varepsilon}}+\left\langle\left(R_{\varepsilon}-\bar{R}\right)_{+}\left|k_{s} s_{\varepsilon}+k_{w} w_{\varepsilon}\right|,\left(R_{\varepsilon}-\bar{R}\right)_{+}\right\rangle_{\Gamma_{\varepsilon}} \leq 0 .
$$

Integrating from 0 to $t$ and using $R_{I} \leq \bar{R}$ leads to

$$
\frac{1}{2}\left\|\left(R_{\varepsilon}-\bar{R}\right)_{+}\right\|_{\Gamma_{\varepsilon}}^{2}+\left\|\left(R_{\varepsilon}-\bar{R}\right)_{+} \sqrt{\left|k_{s} s_{\varepsilon}+k_{w} w_{\varepsilon}\right|}\right\|_{\Gamma_{\varepsilon}, t}^{2} \leq 0 .
$$

We conclude that $R_{\varepsilon}<\bar{R}$ for almost every $x \in \Gamma_{\varepsilon}$ and $t \in[0, T]$.

The required a priori estimates for the other unknowns are proved in Appendix A.1. They are summarized in the following lemma.

LEMma 4.2. The following statements hold:

1. The functions $u_{\varepsilon}, v_{\varepsilon}, s_{\varepsilon}$, and $w_{\varepsilon}$ are nonnegative for almost every $x \in \Omega_{\varepsilon}$, $x \in \Gamma_{\varepsilon}$, respectively, and $t \in[0, T]$.

2. There exists a constant $C>0$, independent of $\varepsilon$, such that

$$
\begin{aligned}
& \left\|u_{\varepsilon}\right\|_{\Omega_{\varepsilon}}^{2}+\left\|v_{\varepsilon}\right\|_{\Omega_{\varepsilon}}^{2}+\varepsilon\left\|s_{\varepsilon}\right\|_{\Gamma_{\varepsilon}}^{2}+\varepsilon\left\|w_{\varepsilon}\right\|_{\Gamma_{\varepsilon}}^{2}+\left\|\nabla u_{\varepsilon}\right\|_{\Omega_{\varepsilon}, t}^{2}+\left\|\nabla v_{\varepsilon}\right\|_{\Omega_{\varepsilon}, t}^{2} \\
& \quad+\varepsilon^{3}\left\|\nabla_{\Gamma} s_{\varepsilon}\right\|_{\Gamma_{\varepsilon}, t}^{2}+\varepsilon^{3}\left\|\nabla_{\Gamma} w_{\varepsilon}\right\|_{\Gamma_{\varepsilon}, t}^{2}+\varepsilon\left\|k_{u} u_{\varepsilon} R_{\varepsilon}-l_{s} s_{\varepsilon}\right\|_{\Gamma_{\varepsilon}, t}^{2} \\
& \quad+\varepsilon\left\|k_{v} v_{\varepsilon} R_{\varepsilon}-l_{w} w_{\varepsilon}\right\|_{\Gamma_{\varepsilon}, t}^{2} \leq C .
\end{aligned}
$$

3. The functions $u_{\varepsilon}, v_{\varepsilon}, s_{\varepsilon}$, and $w_{\varepsilon}$ are bounded independently of $\varepsilon$ almost everywhere in $\Omega_{\varepsilon} \times[0, T]$ and $\Gamma_{\varepsilon} \times[0, T]$, respectively.

4. There exists a $C>0$, independent of $\varepsilon$, such that

$$
\left\|\partial_{t} u_{\varepsilon}\right\|_{L^{2}\left([0, T], H_{0}^{1}\left(\Omega_{\varepsilon}\right)^{\prime}\right)}+\left\|\partial_{t} v_{\varepsilon}\right\|_{L^{2}\left([0, T], H_{0}^{1}\left(\Omega_{\varepsilon}\right)^{\prime}\right)}<C .
$$

Now we know that $u_{\varepsilon}, v_{\varepsilon} \in L^{2}\left([0, T], H^{1}\left(\Omega_{\varepsilon}\right)\right) \cap H^{1}\left([0, T], H_{0}^{1}\left(\Omega_{\varepsilon}\right)^{\prime}\right) \cap L^{\infty}\left(\Omega_{\varepsilon} \times\right.$ $[0, T])$. Using the extension lemma from [18], we extend the functions $u_{\varepsilon}$ and $v_{\varepsilon}$ from $\Omega_{\varepsilon}$ to the whole domain $\Omega$ and know now that $u_{\varepsilon}, v_{\varepsilon} \in L^{2}\left([0, T], H^{1}(\Omega)\right) \cap$ $H^{1}\left([0, T], H_{0}^{1}(\Omega)^{\prime}\right) \cap L^{\infty}(\Omega \times[0, T])$ with bounds independent of $\varepsilon$. Applying Lemma 5.6 from article [17] $u_{\varepsilon}, v_{\varepsilon}$ converge strongly to limit functions $u_{0}, v_{0}$ in $L^{2}([0, T]$, $\left.L^{2}(\Omega)\right)$, respectively. 
We cannot prove strong convergence of the functions $s_{\varepsilon}, w_{\varepsilon}$, and $R_{\varepsilon}$ using extensions to $\Omega$, because they are defined on the $\varepsilon$-dependent manifold $\Gamma_{\varepsilon}$, which has a smaller dimension than $\Omega$. Hence, we use the boundary unfolding operator $\mathcal{T}_{\varepsilon}^{b}$, because it is already defined on a fixed domain $\Omega \times \Gamma$ and show that $\mathcal{T}_{\varepsilon}^{b}\left(s_{\varepsilon}\right), \mathcal{T}_{\varepsilon}^{b}\left(w_{\varepsilon}\right)$, and $\mathcal{T}_{\varepsilon}^{b}\left(R_{\varepsilon}\right)$ are Cauchy-sequences, the proof of which is found in Appendix A.2. This procedure is similar to that in [11], where a nonlinear ordinary differential equation defined on a surface was homogenized.

Lemma $4.3\left(s_{\varepsilon}, w_{\varepsilon}, R_{\varepsilon}\right.$ are Cauchy-sequences). For all $\delta>0$ there exists $\tilde{\varepsilon}>0$ such that for all $0<\varepsilon_{1}, \varepsilon_{2}<\tilde{\varepsilon}$ it holds that

$$
\begin{aligned}
& \left\|\mathcal{T}_{\varepsilon_{1}}^{b}\left(s_{\varepsilon_{1}}\right)-\mathcal{T}_{\varepsilon_{2}}^{b}\left(s_{\varepsilon_{2}}\right)\right\|_{[0, T] \times \Omega \times \Gamma}^{2}+\left\|\mathcal{T}_{\varepsilon_{1}}^{b}\left(w_{\varepsilon_{1}}\right)-\mathcal{T}_{\varepsilon_{2}}^{b}\left(w_{\varepsilon_{2}}\right)\right\|_{[0, T] \times \Omega \times \Gamma}^{2} \\
& \quad+\left\|\mathcal{T}_{\varepsilon_{1}}^{b}\left(R_{\varepsilon_{1}}\right)-\mathcal{T}_{\varepsilon_{2}}^{b}\left(R_{\varepsilon_{2}}\right)\right\|_{[0, T] \times \Omega \times \Gamma}^{2}<\delta .
\end{aligned}
$$

This means that $s_{\varepsilon}, w_{\varepsilon}$, and $R_{\varepsilon}$ are Cauchy-sequences in $L^{2}([0, T] \times \Omega \times \Gamma)$.

Now, we need to ensure that, for every $\varepsilon>0$, there exists a solution of the system of equations (3.2). The following assertion is proved in Appendix B.

Theorem 4.4 (existence of $u_{\varepsilon}, v_{\varepsilon}, s_{\varepsilon}, w_{\varepsilon}$, and $R_{\varepsilon}$ ). For every small $\varepsilon>0$ there exists at least one solution $\left(u_{\varepsilon}, v_{\varepsilon}, s_{\varepsilon}, w_{\varepsilon}, R_{\varepsilon}\right) \in \mathcal{V}_{\mathrm{C}} \times \mathcal{V}_{\mathrm{N}} \times \mathcal{V}\left(\Gamma_{\varepsilon}\right)^{2} \times \mathcal{V}_{R}\left(\Gamma_{\varepsilon}\right)$ of the system (3.2).

Having established these results, we use the results of section 2 to deduce convergence of the solutions of system (3.2) to some limit functions.

TheOrem 4.5. There exist $u_{0}, v_{0} \in L^{2}\left([0, T], H^{1}(\Omega)\right), u_{1}, v_{1} \in L^{2}([0, T] \times$ $\left.\Omega, H_{\#}^{1}\left(Y^{*}\right)\right), s_{0}, w_{0} \in L^{2}\left([0, T] \times \Omega, H_{\#}^{1}(\Gamma)\right)$ and $R_{0} \in L^{2}\left([0, T] \times \Omega, L_{\#}^{2}(\Gamma)\right)$ such that the sequence of solutions $\left(u_{\varepsilon}, v_{\varepsilon}, s_{\varepsilon}, w_{\varepsilon}, R_{\varepsilon}\right)$ of (3.2) converges as $\varepsilon \rightarrow 0$, up to a subsequence, as follows:

1. $\mathcal{T}_{\varepsilon}\left(u_{\varepsilon}\right) \rightarrow u_{0}$ and $\mathcal{T}_{\varepsilon}\left(v_{\varepsilon}\right) \rightarrow v_{0}$ weakly in $L^{2}\left([0, T] \times \Omega, H^{1}\left(Y^{*}\right)\right)$,

2. $\mathcal{T}_{\varepsilon}\left(\nabla_{x} u_{\varepsilon}\right) \rightarrow \nabla_{x} u_{0}+\nabla_{y} u_{1}$ and $\mathcal{T}_{\varepsilon}\left(\nabla_{x} v_{\varepsilon}\right) \rightarrow \nabla_{x} v_{0}+\nabla_{y} v_{1}$ weakly in $L^{2}([0, T] \times$ $\left.\Omega \times Y^{*}\right)$,

3. $\mathcal{T}_{\varepsilon}^{b}\left(s_{\varepsilon}\right) \rightarrow s_{0}$ and $\mathcal{T}_{\varepsilon}^{b}\left(w_{\varepsilon}\right) \rightarrow w_{0}$ weakly in $L^{2}\left([0, T] \times \Omega, H^{1}(\Gamma)\right)$,

4. $\mathcal{T}_{\varepsilon}^{b}\left(R_{\varepsilon}\right) \rightarrow R_{0}$ weakly in $L^{2}([0, T] \times \Omega \times \Gamma)$,

5. $u_{\varepsilon} \rightarrow u_{0}$ and $v_{\varepsilon} \rightarrow v_{0}$ strongly in $L^{2}([0, T] \times \Omega)$,

6. $\mathcal{T}_{\varepsilon}^{b}\left(s_{\varepsilon}\right) \rightarrow s_{0}, \mathcal{T}_{\varepsilon}^{b}\left(w_{\varepsilon}\right) \rightarrow w_{0}$, and $\mathcal{T}_{\varepsilon}^{b}\left(R_{\varepsilon}\right) \rightarrow R_{0}$ strongly in $L^{2}([0, T] \times \Omega \times \Gamma)$.

Proof. Existence of $\left(u_{\varepsilon}, v_{\varepsilon}, s_{\varepsilon}, w_{\varepsilon}, R_{\varepsilon}\right)$ satisfying system (3.2) is provided by Theorem 4.4. The convergences 1-4 follow from the estimates of Lemma 4.2 by applying Theorems 2.2 and 2.9. Furthermore, 5 is deduced by applying Lemma 5.6 of [17] using the estimates of Lemmas 4.1 and 4.2 as described above, while 6 directly follows from Lemma 4.3 .

To find the system of equations satisfied by the limit functions of Theorem 4.5, we pass to the limit in system (3.2) in the next section.

5. Identification of the limit model for the nonlinear carcinogenesis model. First, we consider the $\varepsilon$-limits of the nonlinear terms. Afterward, we derive the complete limit system.

5.1. The nonlinear terms. First, we consider the nonlinear terms $f\left(u_{\varepsilon}\right)$ and $g\left(v_{\varepsilon}\right)$ in the equations for $u_{\varepsilon}$ and $v_{\varepsilon}$, respectively, in the system (3.2). Using the periodic unfolding operator $\mathcal{T}_{\varepsilon}$, the Nemytskii operator for the bounded and continuous functions $f$ and $g$ (see [29]), and the strong convergences of $u_{\varepsilon}$ and $v_{\varepsilon}$ (see Theorem 4.5), it follows that 


$$
\begin{aligned}
f\left(\mathcal{T}_{\varepsilon}\left(u_{\varepsilon}\right)\right) \rightarrow f\left(u_{0}\right) & \text { strongly in } L^{2}\left([0, T] \times \Omega \times Y^{*}\right), \\
g\left(\mathcal{T}_{\varepsilon}\left(v_{\varepsilon}\right)\right) \rightarrow g\left(v_{0}\right) & \text { strongly in } L^{2}\left([0, T] \times \Omega \times Y^{*}\right) .
\end{aligned}
$$

Analogously it holds that

$$
h\left(\mathcal{T}_{\varepsilon}^{b}\left(s_{\varepsilon}\right)\right) \rightarrow h\left(s_{0}\right) \quad \text { strongly in } L^{2}([0, T] \times \Omega \times \Gamma),
$$

since $\mathcal{T}_{\varepsilon}^{b}\left(s_{\varepsilon}\right)$ converges strongly to the function $s_{0} \in L^{2}([0, T] \times \Omega \times \Gamma)$ using Theorem 4.5 and noting that $h$ is continuous and bounded.

Second, we calculate the limits of the nonlinear Robin-boundary terms $k_{u} u_{\varepsilon} R_{\varepsilon}$ and $k_{v} v_{\varepsilon} R_{\varepsilon}$ at the surface of the ER. With Theorem 4.5 we know that $\mathcal{T}_{\varepsilon}^{b}\left(R_{\varepsilon}\right)$ converges strongly to the function $R_{0}$ in $L^{2}([0, T] \times \Omega \times \Gamma)$. Then, we use Lemma 2.5 to deduce that

$$
\lim _{\varepsilon \rightarrow 0} \int_{\Omega \times \Gamma}\left(\mathcal{T}_{\varepsilon}^{b}\left(u_{\varepsilon}\right) \mathcal{T}_{\varepsilon}^{b}\left(R_{\varepsilon}\right)-u_{0} R_{0}\right) \varphi \mathrm{d} \sigma_{y} \mathrm{~d} x=0
$$

and

$$
\lim _{\varepsilon \rightarrow 0} \int_{\Omega \times \Gamma}\left(\mathcal{T}_{\varepsilon}^{b}\left(v_{\varepsilon}\right) \mathcal{T}_{\varepsilon}^{b}\left(R_{\varepsilon}\right)-v_{0} R_{0}\right) \varphi \mathrm{d} \sigma_{y} \mathrm{~d} x=0
$$

for all $\varphi \in C^{\infty}(\Omega \times \Gamma)$.

Now, we perform the limit derivation for the equations $u_{\varepsilon}, v_{\varepsilon}, s_{\varepsilon}, w_{\varepsilon}$, and $R_{\varepsilon}$ and use the just calculated $\varepsilon$-limits of the nonlinear terms.

We test these equations with admissible test functions $\varphi_{\varepsilon} \in C^{\infty}\left(\Omega, C_{\#}^{\infty}(Y)\right)$. As test functions $\varphi_{\varepsilon} \in C^{\infty}\left(\Omega, C_{\#}^{\infty}(Y)\right)$, we choose functions of the form

$$
\varphi_{\varepsilon}\left(x, \frac{x}{\varepsilon}\right)=\varphi_{0}(x)+\varepsilon \varphi_{1}\left(x, \frac{x}{\varepsilon}\right)
$$

with $\left(\varphi_{0}, \varphi_{1}\right) \in C^{\infty}(\Omega) \times C^{\infty}\left(\Omega, C_{\#}^{\infty}(Y)\right)$.

5.2. Calculation of the $\varepsilon$-limits. We use assertion 2 of Lemma 2.4 for the first term on $\Gamma_{\varepsilon}$ in the equation for $u_{\varepsilon}$ in system (3.2) and the integration formula of the periodic unfolding operator,

$$
\begin{aligned}
& \int_{\Omega \times Y^{*}} \partial_{t} \mathcal{T}_{\varepsilon}\left(u_{\varepsilon}\right) \varphi_{\varepsilon} \mathrm{d} y \mathrm{~d} x+D_{u} \int_{\Omega \times Y^{*}} \mathcal{T}_{\varepsilon}\left(\nabla_{x} u_{\varepsilon}\right) \nabla_{x} \varphi_{\varepsilon} \mathrm{d} y \mathrm{~d} x \\
& \quad+\int_{\Omega \times \Gamma} k_{u} \mathcal{T}_{\varepsilon}^{b}\left(u_{\varepsilon}\right) \mathcal{T}_{\varepsilon}^{b}\left(R_{\varepsilon}\right) \varphi_{\varepsilon} \mathrm{d} \sigma_{y} \mathrm{~d} x-\int_{\Omega \times \Gamma} l_{s} \mathcal{T}_{\varepsilon}^{b}\left(s_{\varepsilon}\right) \varphi_{\varepsilon} \mathrm{d} \sigma_{y} \mathrm{~d} x \\
& =-\int_{\Omega \times Y^{*}} f\left(\mathcal{T}_{\varepsilon}\left(u_{\varepsilon}\right)\right) \varphi_{\varepsilon} \mathrm{d} y \mathrm{~d} x .
\end{aligned}
$$

With Theorem 2.2, Lemma 2.5, and the considerations for the nonlinear terms we find for $\varepsilon \rightarrow 0$

$$
\begin{aligned}
& \int_{\Omega \times Y^{*}} \partial_{t} u_{0} \varphi_{0} \mathrm{~d} x \mathrm{~d} y+D_{u} \int_{\Omega \times Y^{*}}\left[\nabla_{x} u_{0}+\nabla_{y} u_{1}\right]\left[\nabla_{x} \varphi_{0}+\nabla_{y} \varphi_{1}\right] \mathrm{d} x \mathrm{~d} y \\
& \quad+\int_{\Omega \times \Gamma}\left(k_{u} u_{0} R_{0}-l_{s} s_{0}\right) \varphi_{0} \mathrm{~d} x \mathrm{~d} \sigma_{y}=-\int_{\Omega \times Y^{*}} f\left(u_{0}\right) \varphi_{0} \mathrm{~d} x \mathrm{~d} y
\end{aligned}
$$

for all $\left(\varphi_{0}, \varphi_{1}\right) \in C^{\infty}(\Omega) \times C^{\infty}\left(\Omega, C_{\#}^{\infty}(Y)\right)$. 
Analogously we obtain for the equation for $v_{\varepsilon}$ and $\varepsilon \rightarrow 0$ that

$$
\begin{aligned}
& \int_{\Omega \times Y^{*}} \partial_{t} v_{0} \varphi_{0} \mathrm{~d} x \mathrm{~d} y+D_{v} \int_{\Omega \times Y^{*}}\left[\nabla_{x} v_{0}+\nabla_{y} v_{1}\right]\left[\nabla_{x} \varphi_{0}+\nabla_{y} \varphi_{1}\right] \mathrm{d} x \mathrm{~d} y \\
& \quad+\int_{\Omega \times \Gamma}\left(k_{v} v_{0} R_{0}-l_{w} w_{0}\right) \varphi_{0} \mathrm{~d} x \mathrm{~d} \sigma_{y}=-\int_{\Omega \times Y^{*}} g\left(v_{0}\right) \varphi_{0} \mathrm{~d} x \mathrm{~d} y
\end{aligned}
$$

for all $\left(\varphi_{0}, \varphi_{1}\right) \in C^{\infty}(\Omega) \times C^{\infty}\left(\Omega, C_{\#}^{\infty}(Y)\right)$.

Again with Lemma 2.5 for the function products and the considerations of the nonlinear terms, we calculate the limit equation for $R_{\varepsilon}$,

$$
\begin{aligned}
& \int_{\Omega \times \Gamma} \partial_{t} \mathcal{T}_{\varepsilon}^{b}\left(R_{\varepsilon}\right) \psi_{\varepsilon} \mathrm{d} \sigma_{y} \mathrm{~d} x+\int_{\Omega \times \Gamma} \mathcal{T}_{\varepsilon}^{b}\left(R_{\varepsilon}\right)\left(k_{u} \mathcal{T}_{\varepsilon}^{b}\left(u_{\varepsilon}\right)+k_{v} \mathcal{T}_{\varepsilon}^{b}\left(v_{\varepsilon}\right)\right. \\
& \left.\quad+l_{s} \mathcal{T}_{\varepsilon}^{b}\left(s_{\varepsilon}\right)+l_{w} \mathcal{T}_{\varepsilon}^{b}\left(w_{\varepsilon}\right)\right) \psi_{\varepsilon} \mathrm{d} \sigma_{y} \mathrm{~d} x \\
& \quad=\int_{\Omega \times \Gamma} \bar{R}\left(l_{s} \mathcal{T}_{\varepsilon}^{b}\left(s_{\varepsilon}\right)+l_{w} \mathcal{T}_{\varepsilon}^{b}\left(w_{\varepsilon}\right)\right) \psi_{\varepsilon} \mathrm{d} \sigma_{y} \mathrm{~d} x .
\end{aligned}
$$

We find for $\varepsilon \rightarrow 0$

$$
\begin{aligned}
& \int_{\Omega \times \Gamma} \partial_{t} R_{0} \psi_{0} \mathrm{~d} x \mathrm{~d} \sigma_{y}+\int_{\Omega \times \Gamma} R_{0}\left(k_{u} u_{0}+k_{v} v_{0}+l_{s} s_{0}+l_{w} w_{0}\right) \psi_{0} \mathrm{~d} x \mathrm{~d} \sigma_{y} \\
& \quad=\int_{\Omega \times \Gamma} \bar{R}\left(l_{s} s_{0}+l_{w} w_{0}\right) \psi_{0} \mathrm{~d} x \mathrm{~d} \sigma_{y}
\end{aligned}
$$

for all $\psi_{0} \in C^{\infty}\left(\Omega, C_{\#}^{\infty}(\Gamma)\right)$.

Now, we calculate the limit equations for $s_{\varepsilon}$ and $w_{\varepsilon}$,

$$
\begin{aligned}
& \int_{\Omega \times \Gamma} \partial_{t} \mathcal{T}_{\varepsilon}^{b}\left(s_{\varepsilon}\right) \psi_{\varepsilon} \mathrm{d} \sigma_{y} \mathrm{~d} x+D_{s} \int_{\Omega \times \Gamma} \nabla_{y} \mathcal{T}_{\varepsilon}^{b}\left(s_{\varepsilon}\right) \nabla_{y} \psi_{\varepsilon} \mathrm{d} \sigma_{y} \mathrm{~d} x \\
& \quad=\int_{\Omega \times \Gamma}\left(k_{u} \mathcal{T}_{\varepsilon}^{b}\left(u_{\varepsilon}\right) \mathcal{T}_{\varepsilon}^{b}\left(R_{\varepsilon}\right)-l_{s} \mathcal{T}_{\varepsilon}^{b}\left(s_{\varepsilon}\right)\right) \psi_{\varepsilon} \mathrm{d} \sigma_{y} \mathrm{~d} x-\int_{\Omega \times \Gamma} \mathcal{T}_{\varepsilon}^{b}\left(h\left(s_{\varepsilon}\right) \psi_{\varepsilon} \mathrm{d} \sigma_{y} \mathrm{~d} x .\right.
\end{aligned}
$$

For this purpose, we use Lemma 2.6 and Theorem 2.9 to deduce for $\varepsilon \rightarrow 0$ that

$$
\begin{aligned}
& \int_{\Omega \times \Gamma} \partial_{t} s_{0} \psi_{0} \mathrm{~d} x \mathrm{~d} \sigma_{y}+D_{s} \int_{\Omega \times \Gamma} \nabla_{y} s_{0} \nabla_{y} \psi_{0} \mathrm{~d} x \mathrm{~d} \sigma_{y} \\
& \quad=\int_{\Omega \times \Gamma}\left(k_{u} u_{0} R_{0}-l_{s} s_{0}\right) \psi_{0} \mathrm{~d} x \mathrm{~d} \sigma_{y}-\int_{\Omega \times \Gamma} h\left(s_{0}\right) \psi_{0} \mathrm{~d} x \mathrm{~d} \sigma_{y}
\end{aligned}
$$

for all $\psi_{0} \in C^{\infty}\left(\Omega, C_{\#}^{\infty}(\Gamma)\right)$. Analogously we get

$$
\begin{aligned}
& \int_{\Omega \times \Gamma} \partial_{t} w_{0} \psi_{0} \mathrm{~d} x \mathrm{~d} \sigma_{y}+D_{w} \int_{\Omega \times \Gamma} \nabla_{\Gamma} w_{0} \nabla_{\Gamma} \psi_{0} \mathrm{~d} x \mathrm{~d} \sigma_{y} \\
& \quad=\int_{\Omega \times \Gamma}\left(k_{v} v_{0} R_{0}-l_{w} w_{0}\right) \psi_{0} \mathrm{~d} x \mathrm{~d} \sigma_{y}+\int_{\Omega \times \Gamma} h\left(s_{0}\right) \psi_{0} \mathrm{~d} x \mathrm{~d} \sigma_{y}
\end{aligned}
$$

for all $\psi_{0} \in C^{\infty}\left(\Omega, C_{\#}^{\infty}(\Gamma)\right)$.

5.3. Identification of $\boldsymbol{u}_{1}(\boldsymbol{x}, \boldsymbol{y}, \boldsymbol{t})$ and $\boldsymbol{v}_{\boldsymbol{1}}(\boldsymbol{x}, \boldsymbol{y}, \boldsymbol{t})$. For (5.1) and (5.2) we obtain the standard cell problem

$$
\begin{aligned}
& \nabla_{y} \cdot\left(e_{j}+\nabla_{y} \mu_{j}\right)=0 \quad \text { in } Y^{*}, \\
& \left(e_{j}+\nabla_{y} \mu_{j}\right) \cdot n=0 \quad \text { on } \partial Y^{*} \text {, }
\end{aligned}
$$

Copyright $@$ by SIAM. Unauthorized reproduction of this article is prohibited. 
where $\mu_{j}$ must be $Y$-periodic for all $j=1, \ldots, n$. This can be found by setting $\varphi_{0}=0$ in (5.1) and is deduced in detail in [19, Chapter 1]. The elements of the diffusion tensors $P^{u}$ and $P^{v}$ are found by setting $\varphi_{1}=0$ and are given by

$$
P_{i j}^{u}=D_{u} \int_{Y^{*}}\left(\delta_{i j}+\partial_{y_{i}} \mu_{j}\right) \mathrm{d} y \quad \text { and } \quad P_{i j}^{v}=D_{v} \int_{Y^{*}}\left(\delta_{i j}+\partial_{y_{i}} \mu_{j}\right) \mathrm{d} y .
$$

5.4. Limit system. Now, we know all equations satisfied by the $\varepsilon$-limits of the solutions of (3.2) as given by Theorem 4.5. For convenience we denote the limit $\left(u_{0}, v_{0}, s_{0}, w_{0}, R_{0}\right)$ by $(u, v, s, w, R)$. We use that $u$ and $v$ are $y$-independent and, before summarizing the homogenized limit problem in the following theorem, we note that every convergent subsequence of the sequence $\left(u_{\varepsilon}, v_{\varepsilon}, s_{\varepsilon}, w_{\varepsilon}, R_{\varepsilon}\right)$ converges to a limit, which satisfies the equations derived above. Because this system of equations has a unique solution, as proved in Theorem 6.1 below, the whole sequence $\left(u_{\varepsilon}, v_{\varepsilon}, s_{\varepsilon}, w_{\varepsilon}, R_{\varepsilon}\right)$ must converge to the solution of this limit problem.

THEOREM 5.1. The homogenized limit problem of model (3.2), satisfied by the limit functions of Theorem 4.5, reads as follows: Find $(u, v, s, w, R) \in \mathcal{V}_{\mathrm{C}}(\Omega) \times \mathcal{V}_{\mathrm{N}}(\Omega) \times$ $\mathcal{V}(\Omega, \Gamma)^{2} \times \mathcal{V}_{R}(\Omega, \Gamma)$ satisfying

$$
(u(0), v(0), s(0), w(0), R)=\left(u_{I}, v_{I}, s_{I}, w_{I}, R_{I}\right)
$$

and

$$
\begin{aligned}
\left|Y^{*}\right|\left(\partial_{t} u, \varphi_{1}\right)_{\Omega}+\left(P^{u} \nabla u, \nabla \varphi_{1}\right)_{\Omega}+\left(k_{u} u R-l_{s} s, \varphi_{1}\right)_{\Omega \times \Gamma} & =-\left|Y^{*}\right|\left(f(u), \varphi_{1}\right)_{\Omega}, \\
\left|Y^{*}\right|\left(\partial_{t} v, \varphi_{2}\right)_{\Omega}+\left(P^{v} \nabla v, \nabla \varphi_{2}\right)_{\Omega}+\left(k_{v} v R-l_{w} w, \varphi_{2}\right)_{\Omega \times \Gamma} & =-\left|Y^{*}\right|\left(g(v), \varphi_{2}\right)_{\Omega}, \\
\left(\partial_{t} s, \psi\right)_{\Omega \times \Gamma}+D_{s}\left(\nabla_{\Gamma} s, \nabla_{\Gamma} \psi\right)_{\Omega \times \Gamma}-\left(k_{u} u R-l_{s} s, \psi\right)_{\Omega \times \Gamma} & =-(h(s), \psi)_{\Omega \times \Gamma}, \\
\left(\partial_{t} w, \psi\right)_{\Omega \times \Gamma}+D_{w}\left(\nabla_{\Gamma} w, \nabla_{\Gamma} \psi\right)_{\Omega \times \Gamma}-\left(k_{v} v R-l_{w} w, \psi\right)_{\Omega \times \Gamma} & =(h(s), \psi)_{\Omega \times \Gamma}, \\
\left(\partial_{t} R, \psi\right)_{\Omega \times \Gamma}+\left(R\left(k_{u} u+k_{v} v+l_{s} s+l_{w} w\right), \psi\right)_{\Omega \times \Gamma} & =\left(\bar{R}\left(l_{s} s+l_{w} w\right), \psi\right)_{\Omega \times \Gamma},
\end{aligned}
$$

for all $\left(\varphi_{1}, \varphi_{2}, \psi\right) \in V_{\mathrm{C} 0}(\Omega) \times V_{\mathrm{N}}(\Omega) \times V(\Omega, \Gamma)$, where the effective diffusion tensors $P^{u}$ and $P^{v}$ are given by (5.4).

For the reader's convenience, we also state the strong form of the limit system (5.5): Find $(u, v, s, w, R)$ satisfying (5.5a) as well as

$$
\begin{aligned}
\left|Y^{*}\right| \partial_{t} u-\nabla \cdot P^{u} \nabla u+\int_{\Gamma}\left(k_{u} u R-l_{s} s\right) \mathrm{d} \sigma_{y} & =-\left|Y^{*}\right| f(u) & & \text { in } \Omega, \\
\left|Y^{*}\right| \partial_{t} v-\nabla \cdot P^{v} \nabla v+\int_{\Gamma}\left(k_{v} v R-l_{w} w\right) \mathrm{d} \sigma_{y} & =-\left|Y^{*}\right| g(v) & & \text { in } \Omega, \\
\partial_{t} s+\nabla_{\Gamma} \cdot\left(D_{s} \nabla_{\Gamma} s\right)-\left(k_{u} u R-l_{s} s\right) & =-h(s) & & \text { in } \Omega \times \Gamma, \\
\partial_{t} w-\nabla_{\Gamma} \cdot\left(D_{w} \nabla_{\Gamma} w\right)-\left(k_{v} v R-l_{w} w\right) & =h(s) & & \text { in } \Omega \times \Gamma, \\
\partial_{t} R+R\left(k_{u} u+k_{v} v+l_{s} s+l_{w} w\right) & =\bar{R}\left(l_{s} s+l_{w} w\right) & & \text { in } \Omega \times \Gamma .
\end{aligned}
$$

and

$$
\begin{aligned}
u & =u_{\text {Boundary }} & & \text { on } \Gamma_{\mathrm{C}}, \\
-P^{u} \nabla u \cdot n & =0 & & \text { on } \Gamma_{\mathrm{N}}, \\
-P^{v} \nabla v \cdot n & =0 & & \text { on } \Gamma_{\mathrm{C}}, \\
v & =0 & & \text { on } \Gamma_{\mathrm{N}} .
\end{aligned}
$$

To conclude the analysis, we show the uniqueness of the solution of (5.5) in section 6 .

Copyright $@$ by SIAM. Unauthorized reproduction of this article is prohibited. 
6. Uniqueness of the limit model. In this section we show that the solution of system (5.5) is unique.

THEOREM 6.1. There is at most one solution of problem (5.5).

Proof. To prove uniqueness of the homogenized limit model, we need to show uniqueness of the cell problem (5.3) and the macroscopic system of (5.5).

Uniqueness up to a constant of the solution of the cell problem (5.3) is proved in [19] and it is left to show uniqueness of the macroscopic system of equations. Let us suppose that there exist two solutions $\left(u_{1}, v_{1}, s_{1}, w_{1}, R_{1}\right)$ and $\left(u_{2}, v_{2}, s_{2}, w_{2}, R_{2}\right)$ of the weak problem (5.5) with the same given initial values. We want to show that $\left(u_{1}, v_{1}, s_{1}, w_{1}, R_{1}\right)=\left(u_{2}, v_{2}, s_{2}, w_{2}, R_{2}\right)$ almost everywhere.

Now, we take the equations for $u_{1}$ and $u_{2}$, subtract them from each other, and test with $\varphi=u_{1}-u_{2}$. Integration from 0 to $t$ yields

$$
\begin{aligned}
|Y| \frac{1}{2}\left\|u_{1}-u_{2}\right\|_{\Omega}^{2}+\left\|\sqrt{P^{u}} \nabla\left(u_{1}-u_{2}\right)\right\|_{\Omega \times[0, t]}^{2} \\
\quad+\left(k_{u}\left(u_{1} R_{1}-u_{2} R_{2}\right)-l_{s}\left(s_{1}-s_{2}\right), u_{1}-u_{2}\right)_{\Omega \times \Gamma} \\
=-|Y|\left(f\left(u_{1}\right)-f\left(u_{2}\right), u_{1}-u_{2}\right)_{\Omega} \leq 0
\end{aligned}
$$

since $f$ is monotone. Adding and subtracting $u_{1} R_{2}$ in the third term, we obtain with the binomial theorem that

$$
\begin{aligned}
|Y| & \frac{1}{2}\left\|u_{1}-u_{2}\right\|_{\Omega}^{2}+\left\|\sqrt{P^{u}} \nabla\left(u_{1}-u_{2}\right)\right\|_{\Omega, t}^{2} \\
\leq & |\Gamma|\left(k_{u}\|u\|_{L^{\infty}}+k_{u} \bar{R}+l_{s}\right)\left\|u_{1}-u_{2}\right\|_{\Omega, t}^{2}+k_{u}\|u\|_{L^{\infty}}\left\|R_{1}-R_{2}\right\|_{\Omega \times \Gamma, t}^{2} \\
& \quad+l_{s}\left\|s_{1}-s_{2}\right\|_{\Omega \times \Gamma, t}^{2} .
\end{aligned}
$$

Analogously, we find similar estimations for the equations for $v, s, w$, and $R$. We add them up and obtain

$$
\begin{aligned}
& \left\|u_{1}-u_{2}\right\|_{\Omega}^{2}+\left\|v_{1}-v_{2}\right\|_{\Omega}^{2}+\left\|s_{1}-s_{2}\right\|_{\Omega \times \Gamma}^{2}+\left\|w_{1}-w_{2}\right\|_{\Omega \times \Gamma}^{2}+\left\|R_{1}-R_{2}\right\|_{\Omega \times \Gamma}^{2} \\
& \leq c_{1}\left(\left\|u_{1}-u_{2}\right\|_{\Omega, t}^{2}+\left\|v_{1}-v_{2}\right\|_{\Omega, t}^{2}+\left\|s_{1}-s_{2}\right\|_{\Omega \times \Gamma, t}^{2}+\left\|w_{1}-w_{2}\right\|_{\Omega \times \Gamma, t}^{2}\right. \\
& \left.\quad+\left\|R_{1}-R_{2}\right\|_{\Omega \times \Gamma, t}^{2}\right)
\end{aligned}
$$

for a constant $c_{1}>0$.

Gronwall's lemma implies

$$
\left\|u_{1}-u_{2}\right\|_{\Omega}^{2}+\left\|v_{1}-v_{2}\right\|_{\Omega}^{2}+\left\|s_{1}-s_{2}\right\|_{\Omega \times \Gamma}^{2}+\left\|w_{1}-w_{2}\right\|_{\Omega \times \Gamma}^{2}+\left\|R_{1}-R_{2}\right\|_{\Omega \times \Gamma}^{2} \leq 0
$$

and we obtain that $u_{1}=u_{2}$ and $v_{1}=v_{2}$ almost everywhere in $\Omega$ and $s_{1}=s_{2}, w_{1}=w_{2}$ and $R_{1}=R_{2}$ almost everywhere in $\Omega \times \Gamma$ and for almost every $t \in[0, T]$.

7. Conclusions. The limit model (5.5) (or, in its strong form, (5.6)) for carcinogenesis obtained in the homogenization process is of distributed-microstructure type. It consists of two partial differential equations involving global diffusion for the two species defined in the cytosol coupled to two partial differential equations involving local diffusion on the surface of the endoplasmic reticulum in a representative unit cell attached to each macroscopic point in space. Moreover, the number of free receptors in each representative unit cell is accounted for by an ordinary differential equation for this quantity. All parameters of the homogenized model are explicitly related to those of the microscopic model. In the future, it would be of great interest to test the model qualitatively and quantitatively, for which corresponding experimental data is 
required. In this context, it might be useful to look into different scalings as well (as in $[16,26]$ ) or to include more complex exchange mechanisms through membranes (as in $[31])$.

From a homogenization point of view, the compactness result in Theorem 2.9 is worth highlighting as this result should be useful whenever systems involving slow diffusion on hypersurfaces are to be homogenized using the periodic unfolding method.

\section{Appendix A. Estimates.}

\section{A.1. A priori estimates.}

Lemma A.1 (positivity). The functions $u_{\varepsilon}, v_{\varepsilon}, s_{\varepsilon}$, and $w_{\varepsilon}$ are nonnegative for almost every $x \in \Omega_{\varepsilon}, x \in \Gamma_{\varepsilon}$, respectively, and $t \in[0, T]$.

Proof. We start with the equations for $u_{\varepsilon}$ and $s_{\varepsilon}$ and test the weak formulation with $u_{\varepsilon_{-}}$and $s_{\varepsilon_{-}}$, respectively, and add them up,

$$
\begin{aligned}
& \left(\partial_{t} u_{\varepsilon}, u_{\varepsilon-}\right)_{\Omega_{\varepsilon}}+\varepsilon\left\langle\partial_{t} s_{\varepsilon}, s_{\varepsilon-}\right\rangle_{\Gamma_{\varepsilon}}+D_{u}\left(\nabla u_{\varepsilon}, \nabla u_{\varepsilon-}\right)_{\Omega_{\varepsilon}}+D_{s} \varepsilon^{3}\left\langle\nabla_{\Gamma} s_{\varepsilon}, \nabla_{\Gamma} s_{\varepsilon-}\right\rangle_{\Gamma_{\varepsilon}} \\
& \quad+\varepsilon\left\langle k_{u} u_{\varepsilon} R_{\varepsilon}-l_{s} s_{\varepsilon}, u_{\varepsilon-}-s_{\varepsilon-}\right\rangle_{\Gamma_{\varepsilon}}=-\left(f\left(u_{\varepsilon}\right), u_{\varepsilon-}\right)_{\Omega_{\varepsilon}}-\varepsilon\left\langle h\left(s_{\varepsilon}\right), s_{\varepsilon-}\right\rangle_{\Gamma_{\varepsilon}}=0 .
\end{aligned}
$$

Multiplying with -1 leads to

$$
\begin{aligned}
\left(\partial_{t} u_{\varepsilon-}, u_{\varepsilon-}\right) \Omega_{\varepsilon}+\varepsilon\left\langle\partial_{t} s_{\varepsilon-}, s_{\varepsilon-}\right\rangle_{\Gamma_{\varepsilon}}+D_{u}\left(\nabla u_{\varepsilon-}, \nabla u_{\varepsilon-}\right)_{\Omega_{\varepsilon}}+D_{s} \varepsilon^{3}\left\langle\nabla_{\Gamma} s_{\varepsilon-}, \nabla_{\Gamma} s_{\varepsilon-}\right\rangle_{\Gamma_{\varepsilon}} & \\
& +\varepsilon\left\langle k_{u} u_{\varepsilon-} R_{\varepsilon}, u_{\varepsilon-}\right\rangle_{\Gamma_{\varepsilon}}+\varepsilon\left\langle l_{s} s_{\varepsilon-}, s_{\varepsilon-}\right\rangle_{\Gamma_{\varepsilon}} \\
= & \underbrace{-\varepsilon\left\langle k_{u} u_{\varepsilon+} R_{\varepsilon}, s_{\varepsilon-}\right\rangle_{\Gamma_{\varepsilon}}-\varepsilon\left\langle l_{s} s_{\varepsilon+}, u_{\varepsilon-}\right\rangle_{\Gamma_{\varepsilon}}}_{\leq 0}+\varepsilon\left\langle k_{u} u_{\varepsilon-} R_{\varepsilon}, s_{\varepsilon-}\right\rangle_{\Gamma_{\varepsilon}}+\varepsilon\left\langle l_{s} s_{\varepsilon-}, u_{\varepsilon-}\right\rangle_{\Gamma_{\varepsilon}} .
\end{aligned}
$$

We drop the negative term on the right-hand side and integrate from 0 to $t$. The trace inequality and the Cauchy-Schwarz inequality yield

$$
\begin{aligned}
& \frac{1}{2}\left\|u_{\varepsilon-}\right\|_{\Omega_{\varepsilon}}^{2}+\frac{1}{2} \varepsilon\left\|s_{\varepsilon-}\right\|_{\Gamma_{\varepsilon}}^{2}+\left(D_{u}-\varepsilon^{2}\left(l_{s}+k_{u} \bar{R}\right) c_{0}\right)\left\|\nabla u_{\varepsilon-}\right\|_{\Omega_{\varepsilon}, t}^{2}+D_{s} \varepsilon^{3}\left\|\nabla_{\Gamma} s_{\varepsilon-}\right\|_{\Gamma_{\varepsilon}, t}^{2} \\
& \quad+k_{u} \varepsilon\left\|u_{\varepsilon-} \sqrt{R_{\varepsilon}}\right\|_{\Gamma_{\varepsilon}, t}^{2}+\varepsilon l_{s}\left\|s_{\varepsilon-}\right\|_{\Gamma_{\varepsilon}, t}^{2} \leq\left(l_{s}+k_{u} \bar{R}\right)\left(\varepsilon\left\|s_{\varepsilon-}\right\|_{\Gamma_{\varepsilon}, t}^{2}+c_{0}\left\|u_{\varepsilon-}\right\|_{\Omega_{\varepsilon}, t}^{2}\right) .
\end{aligned}
$$

After merging the constants, for $\varepsilon$ small and with Gronwall's lemma we deduce that $\left\|u_{\varepsilon-}\right\|_{\Omega_{\varepsilon}}^{2}+\left\|s_{\varepsilon-}\right\|_{\Gamma_{\varepsilon}}^{2} \leq 0$ and therefore $u_{\varepsilon}$ and $s_{\varepsilon}$ are greater than or equal to zero for almost every $x \in \Omega_{\varepsilon}$ or $x \in \Gamma_{\varepsilon}$ and $t \in[0, T]$. With similar estimations we also obtain that $v_{\varepsilon}$ and $w_{\varepsilon}$ are nonnegative for almost every $x \in \Omega_{\varepsilon}$ or $x \in \Gamma_{\varepsilon}$ and $t \in[0, T]$.

Lemma A.2 (boundedness in $L^{2}$ ). There exists a constant $C>0$, independent of $\varepsilon$, such that

$$
\begin{aligned}
& \left\|u_{\varepsilon}\right\|_{\Omega_{\varepsilon}}^{2}+\left\|v_{\varepsilon}\right\|_{\Omega_{\varepsilon}}^{2}+\varepsilon\left\|s_{\varepsilon}\right\|_{\Gamma_{\varepsilon}}^{2}+\varepsilon\left\|w_{\varepsilon}\right\|_{\Gamma_{\varepsilon}}^{2} \\
& \quad+\left\|\nabla u_{\varepsilon}\right\|_{\Omega_{\varepsilon}, t}^{2}+\left\|\nabla v_{\varepsilon}\right\|_{\Omega_{\varepsilon}, t}^{2}+\varepsilon^{3}\left\|\nabla_{\Gamma} s_{\varepsilon}\right\|_{\Gamma_{\varepsilon}, t}^{2}+\varepsilon^{3}\left\|\nabla_{\Gamma} w_{\varepsilon}\right\|_{\Gamma_{\varepsilon}, t}^{2} \\
& \quad+\varepsilon\left\|k_{u} u_{\varepsilon} R_{\varepsilon}-l_{s} s_{\varepsilon}\right\|_{\Gamma_{\varepsilon}, t}^{2}+\varepsilon\left\|k_{v} v_{\varepsilon} R_{\varepsilon}-l_{w} w_{\varepsilon}\right\|_{\Gamma_{\varepsilon}, t}^{2} \leq C .
\end{aligned}
$$

Proof. We perform the proof for $u_{\varepsilon}$ and $s_{\varepsilon}$. The estimations for $v_{\varepsilon}$ and $w_{\varepsilon}$ are analogous. For that purpose we test the weak formulations for $u_{\varepsilon}$ and $s_{\varepsilon}$ with $k_{u} \bar{R} u_{\varepsilon}$ and $l_{s} s_{\varepsilon}$, respectively,

$$
\begin{aligned}
& k_{u} \bar{R}\left(\partial_{t} u_{\varepsilon}, u_{\varepsilon}\right)_{\Omega_{\varepsilon}}+\varepsilon l_{s}\left\langle\partial_{t} s_{\varepsilon}, s_{\varepsilon}\right\rangle_{\Gamma_{\varepsilon}}+D_{u} k_{u} \bar{R}\left(\nabla u_{\varepsilon}, \nabla u_{\varepsilon}\right)_{\Omega_{\varepsilon}}+\varepsilon^{3} D_{s} l_{s}\left\langle\nabla_{\Gamma} s_{\varepsilon}, \nabla_{\Gamma} s_{\varepsilon}\right\rangle_{\Gamma_{\varepsilon}} \\
& \quad+\varepsilon\left\langle R_{\varepsilon} u_{\varepsilon} k_{u}-l_{s} s_{\varepsilon}, k_{u} \bar{R} u_{\varepsilon}-l_{s} s_{\varepsilon}\right\rangle_{\Gamma_{\varepsilon}}=-k_{u} \bar{R}\left(f\left(u_{\varepsilon}\right), u_{\varepsilon}\right)_{\Omega_{\varepsilon}}-\varepsilon l_{s}\left\langle h\left(s_{\varepsilon}\right), s_{\varepsilon}\right\rangle_{\Gamma_{\varepsilon}} \leq 0 .
\end{aligned}
$$

Copyright $@$ ㅇ by SIAM. Unauthorized reproduction of this article is prohibited. 
We add $\varepsilon\left\langle k_{u} R_{\varepsilon} u_{\varepsilon}-l_{s} s_{\varepsilon}, R_{\varepsilon} k_{u} u_{\varepsilon}-\bar{R} k_{u} u_{\varepsilon}\right\rangle_{\Gamma_{\varepsilon}}$ on the left-hand and on the right-hand side and compute

$$
\begin{aligned}
& k_{u} \bar{R}\left(\partial_{t} u_{\varepsilon}, u_{\varepsilon}\right)_{\Omega_{\varepsilon}}+\varepsilon l_{s}\left\langle\partial_{t} s_{\varepsilon}, s_{\varepsilon}\right\rangle_{\Gamma_{\varepsilon}}+D_{u} k_{u} \bar{R}\left(\nabla u_{\varepsilon}, \nabla u_{\varepsilon}\right)_{\Omega_{\varepsilon}}+\varepsilon^{3} D_{s} l_{s}\left\langle\nabla_{\Gamma} s_{\varepsilon}, \nabla_{\Gamma} s_{\varepsilon}\right\rangle_{\Gamma_{\varepsilon}} \\
& \quad+\varepsilon\left\langle R_{\varepsilon} u_{\varepsilon} k_{u}-l_{s} s_{\varepsilon}, k_{u} R u_{\varepsilon}-l_{s} s_{\varepsilon}\right\rangle_{\Gamma_{\varepsilon}} \leq \varepsilon\left\langle k_{u} R_{\varepsilon} u_{\varepsilon}-l_{s} s_{\varepsilon}, R_{\varepsilon} k_{u} u_{\varepsilon}-\bar{R} k_{u} u_{\varepsilon}\right\rangle_{\Gamma_{\varepsilon}} .
\end{aligned}
$$

With integration from 0 to $t$ we obtain

$$
\begin{aligned}
\frac{1}{2} k_{u} \bar{R}\left\|u_{\varepsilon}\right\|_{\Omega_{\varepsilon}}^{2}+D_{u} k_{u} \bar{R}\left\|\nabla u_{\varepsilon}\right\|_{\Omega_{\varepsilon}, t}^{2}+\frac{1}{2} \varepsilon l_{s}\left\|s_{\varepsilon}\right\|_{\Gamma_{\varepsilon}}^{2} \\
\quad+\varepsilon^{3} D_{s} l_{s}\left\|\nabla_{\Gamma} s_{\varepsilon}\right\|_{\Gamma_{\varepsilon}, t}^{2}+\varepsilon\left\|k_{u} R_{\varepsilon} u_{\varepsilon}-l_{s} s_{\varepsilon}\right\|_{\Gamma_{\varepsilon}, t}^{2} \\
=\varepsilon\left\langle k_{u} R_{\varepsilon} u_{\varepsilon}-l_{s} s_{\varepsilon}, k_{u} u_{\varepsilon}\left(R_{\varepsilon}-\bar{R}\right)\right\rangle_{\Gamma_{\varepsilon}, t}+\underbrace{\frac{1}{2} k_{u} \bar{R}\left\|u_{\varepsilon}(0)\right\|_{\Omega_{\varepsilon}}^{2}+\frac{1}{2} \varepsilon l_{s}\left\|s_{\varepsilon}(0)\right\|_{\Gamma_{\varepsilon}}^{2}}_{=c_{1}} .
\end{aligned}
$$

Using the binomial theorem for a $\lambda>0$ and the trace inequality gives

$$
\begin{aligned}
\frac{1}{2} k_{u} \bar{R}\left\|u_{\varepsilon}\right\|_{\Omega_{\varepsilon}}^{2}+D_{u} k_{u} \bar{R}\left\|\nabla u_{\varepsilon}\right\|_{\Omega_{\varepsilon}, t}^{2}+\frac{1}{2} \varepsilon l_{s}\left\|s_{\varepsilon}\right\|_{\Gamma_{\varepsilon}}^{2} & \\
& +\varepsilon^{3} D_{s} l_{s}\left\|\nabla_{\Gamma} s_{\varepsilon}\right\|_{\Gamma_{\varepsilon}, t}^{2}+\varepsilon\left\|R_{\varepsilon} u_{\varepsilon} k_{u}-l_{s} s_{\varepsilon}\right\|_{\Gamma_{\varepsilon}, t}^{2} \\
\leq & c_{1}+\frac{1}{2 \lambda} \varepsilon\left\|k_{u} R_{\varepsilon} u_{\varepsilon}-l_{s} s_{\varepsilon}\right\|_{\Gamma_{\varepsilon}, t}^{2}+\frac{\lambda}{2} \varepsilon k_{u}^{2} \bar{R}^{2}\left\|u_{\varepsilon}\right\|_{\Gamma_{\varepsilon}, t}^{2} \\
\leq & c_{1}+\frac{1}{2 \lambda} \varepsilon\left\|k_{u} R_{\varepsilon} u_{\varepsilon}-l_{s} s_{\varepsilon}\right\|_{\Gamma_{\varepsilon}, t}^{2}+\frac{\lambda}{2} k_{u}^{2} \bar{R}^{2} c_{0}\left(\left\|u_{\varepsilon}\right\|_{\Omega_{\varepsilon}, t}^{2}+\varepsilon^{2}\left\|\nabla u_{\varepsilon}\right\|_{\Omega_{\varepsilon}, t}^{2}\right) .
\end{aligned}
$$

Hence,

$$
\begin{aligned}
& \frac{1}{2} k_{u} \bar{R}\left\|u_{\varepsilon}\right\|_{\Omega_{\varepsilon}}^{2}+\left(D_{u} k_{u} \bar{R}-\frac{\lambda}{2} k_{u}^{2} \bar{R}^{2} c_{0} \varepsilon^{2}\right)\left\|\nabla u_{\varepsilon}\right\|_{\Omega_{\varepsilon}, t}^{2} \\
& \quad+\frac{1}{2} \varepsilon l_{s}\left\|s_{\varepsilon}\right\|_{\Gamma_{\varepsilon}}^{2}+\varepsilon^{3} D_{s} l_{s}\left\|\nabla_{\Gamma} s_{\varepsilon}\right\|_{\Gamma_{\varepsilon}, t}^{2}+\varepsilon\left(1-\frac{1}{2 \lambda}\right)\left\|R_{\varepsilon} u_{\varepsilon} k_{u}-l_{s} s_{\varepsilon}\right\|_{\Gamma_{\varepsilon}, t}^{2} \\
& \leq c_{1}+\frac{\lambda}{2} k_{u}^{2} \bar{R}^{2} c_{0}\left\|u_{\varepsilon}\right\|_{\Omega_{\varepsilon}, t}^{2} .
\end{aligned}
$$

With $\lambda>\frac{1}{2}$ and $\varepsilon$ small, we can merge the constants and use Gronwall's lemma to deduce the assertion.

To prove strong convergence it is necessary to show that $u_{\varepsilon}, v_{\varepsilon} \in L^{\infty}\left(\Omega_{\varepsilon}\right)$ and $s_{\varepsilon}, w_{\varepsilon} \in L^{\infty}\left(\Gamma_{\varepsilon}\right)$. We already know that $u_{\varepsilon}, v_{\varepsilon}, s_{\varepsilon}, w_{\varepsilon}$ are nonnegative and, hence, bounded from below. It is left to show boundedness from above. We make use of the fact that $R_{\varepsilon} \in L^{\infty}\left(\Gamma_{\varepsilon}\right)$, which we established in Lemma 4.1.

Lemma A.3 (boundedness in $L^{\infty}$ ). The functions $u_{\varepsilon}, v_{\varepsilon}, s_{\varepsilon}$, and $w_{\varepsilon}$ are bounded independently of $\varepsilon$ almost everywhere in $\Omega_{\varepsilon} \times[0, T]$ and $\Gamma_{\varepsilon} \times[0, T]$, respectively.

Proof. Let $M(t)=\max \left\{\left\|u_{I}\right\|_{L^{\infty}\left(\Omega_{\varepsilon}\right)},\left\|v_{I}\right\|_{L^{\infty}\left(\Omega_{\varepsilon}\right)},\left\|s_{I}\right\|_{L^{\infty}\left(\Gamma_{\varepsilon}\right)},\left\|w_{I}\right\|_{L^{\infty}\left(\Gamma_{\varepsilon}\right)}\right\} e^{k t}$ for a constant $k \in \mathbf{R}$. The function $M$ exists because the initial conditions are bounded. At first we prove the assertion for $u_{\varepsilon}$ and $s_{\varepsilon}$. We test the weak formulation for $u_{\varepsilon}, s_{\varepsilon}$ with $\left(\bar{R} k_{u} u_{\varepsilon}-M\right)_{+}$and $\left(l_{s} s_{\varepsilon}-M\right)_{+}$, respectively. Then, we add the two equations,

Copyright $@$ ㅇ by SIAM. Unauthorized reproduction of this article is prohibited. 


$$
\begin{aligned}
\left(\partial_{t} u_{\varepsilon},\right. & \left.\left(\bar{R} k_{u} u_{\varepsilon}-M\right)_{+}\right)_{\Omega_{\varepsilon}}+\varepsilon\left\langle\partial_{t} s_{\varepsilon},\left(l_{s} s_{\varepsilon}-M\right)_{+}\right\rangle_{\Gamma_{\varepsilon}}+\left(D_{u} \nabla u_{\varepsilon}, \nabla\left(\bar{R} k_{u} u_{\varepsilon}-M\right)_{+}\right)_{\Omega_{\varepsilon}} \\
& +\varepsilon^{3}\left\langle D_{s} \nabla_{\Gamma} s_{\varepsilon}, \nabla_{\Gamma}\left(l_{s} s_{\varepsilon}-M\right)_{+}\right\rangle_{\Gamma_{\varepsilon}}+\varepsilon\left\langle k_{u} R_{\varepsilon} u_{\varepsilon}-l_{s} s_{\varepsilon},\left(\bar{R} k_{u} u_{\varepsilon}-M\right)_{+}\right\rangle_{\Gamma_{\varepsilon}} \\
= & \underbrace{\left(-f\left(u_{\varepsilon}\right),\left(\bar{R} k_{u} u_{\varepsilon}-M\right)_{+}\right)_{\Omega_{\varepsilon}}}_{\leq 0}+\varepsilon\left\langle k_{u} R_{\varepsilon} u_{\varepsilon}-l_{s} s_{\varepsilon},\left(l_{s} s_{\varepsilon}-M\right)_{+}\right\rangle_{\Gamma_{\varepsilon}} \\
& +\underbrace{\left\langle-h\left(s_{\varepsilon}\right),\left(l_{s} s_{\varepsilon}-M\right)_{+}\right\rangle_{\Gamma_{\varepsilon}}}_{\leq 0}
\end{aligned}
$$

and estimate

$$
\begin{aligned}
& \frac{1}{\bar{R} k_{u}}\left(\partial_{t}\left(\bar{R} k_{u} u_{\varepsilon}-M\right)_{+},\left(\bar{R} k_{u} u_{\varepsilon}-M\right)_{+}\right)_{\Omega_{\varepsilon}}+\frac{1}{l_{s}} \varepsilon\left\langle\partial_{t}\left(l_{s} s_{\varepsilon}-M\right)_{+},\left(l_{s} s_{\varepsilon}-M\right)_{+}\right\rangle_{\Gamma_{\varepsilon}} \\
& \quad+\frac{D_{u}}{\bar{R} k_{u}}\left\|\nabla\left(\bar{R} k_{u} u_{\varepsilon}-M\right)_{+}\right\|_{\Omega_{\varepsilon}}^{2}+\frac{D_{s}}{l_{s}} \varepsilon^{3}\left\|\nabla_{\Gamma}\left(l_{s} s_{\varepsilon}-M\right)_{+}\right\|_{\Gamma_{\varepsilon}}^{2} \\
& \quad+\varepsilon\left\langle k_{u} R_{\varepsilon} u_{\varepsilon}-l_{s} s_{\varepsilon},\left(\bar{R} k_{u} u_{\varepsilon}-M\right)_{+}-\left(l_{s} s_{\varepsilon}-M\right)_{+}\right\rangle_{\Gamma_{\varepsilon}} \\
& \leq-\frac{1}{\bar{R} k_{u}}\left(M k,\left(\bar{R} k_{u} u_{\varepsilon}-M\right)_{+}\right)_{\Omega_{\varepsilon}}-\frac{1}{l_{s}} \varepsilon\left\langle M k,\left(l_{s} s_{\varepsilon}-M\right)_{+}\right\rangle_{\Gamma_{\varepsilon}} .
\end{aligned}
$$

We add $\varepsilon\left\langle k_{u} \bar{R} u_{\varepsilon}-k_{u} R_{\varepsilon} u_{\varepsilon},\left(\bar{R} k_{u} u_{\varepsilon}-M\right)_{+}-\left(l_{s} s_{\varepsilon}-M\right)_{+}\right\rangle_{\Gamma_{\varepsilon}}$ on each side of the inequality, integrate from 0 to $t$, and use the binomial theorem for any $\lambda>0$

$$
\begin{aligned}
& \frac{1}{2 \bar{R} k_{u}}\left\|\left(\bar{R} k_{u} u_{\varepsilon}-M\right)_{+}\right\|_{\Omega_{\varepsilon}}^{2}+\frac{1}{2 l_{s}} \varepsilon\left\|\left(l_{s} s_{\varepsilon}-M\right)_{+}\right\|_{\Gamma_{\varepsilon}}^{2}+\frac{D_{u}}{\bar{R} k_{u}}\left\|\nabla\left(\bar{R} k_{u} u_{\varepsilon}-M\right)_{+}\right\|_{\Omega_{\varepsilon}, t}^{2} \\
& \quad+\frac{D_{s}}{l_{s}} \varepsilon^{3}\left\|\nabla_{\Gamma}\left(l_{s} s_{\varepsilon}-M\right)_{+}\right\|_{\Gamma_{\varepsilon}, t}^{2}+\varepsilon\left\|\left(\bar{R} k_{u} u_{\varepsilon}-M\right)_{+}-\left(l_{s} s_{\varepsilon}-M\right)_{+}\right\|_{\Gamma_{\varepsilon}, t}^{2} \\
& \leq \lambda \bar{R}^{2} k_{u}^{2} \varepsilon\left\|u_{\varepsilon}\right\|_{\Gamma_{\varepsilon}}^{2}+\frac{1}{2 \lambda} \varepsilon\left\|\left(\bar{R} k_{u} u_{\varepsilon}-M\right)_{+}-\left(l_{s} s_{\varepsilon}-M\right)_{+}\right\|_{\Gamma_{\varepsilon}, t}^{2} \\
& \quad-\frac{1}{\bar{R} k_{u}}\left(M k,\left(\bar{R} k_{u} u_{\varepsilon}-M\right)_{+}\right)_{\Omega_{\varepsilon}, t}-\frac{1}{l_{s}} \varepsilon\left\langle M k,\left(l_{s} s_{\varepsilon}-M\right)_{+}\right\rangle_{\Gamma_{\varepsilon}, t} .
\end{aligned}
$$

Simplifying further yields

$$
\begin{aligned}
& \frac{1}{2 \bar{R} k_{u}}\left\|\left(\bar{R} k_{u} u_{\varepsilon}-M\right)_{+}\right\|_{\Omega_{\varepsilon}}^{2}+\frac{1}{2 l_{s}} \varepsilon\left\|\left(l_{s} s_{\varepsilon}-M\right)_{+}\right\|_{\Gamma_{\varepsilon}}^{2}+\frac{D_{u}}{\bar{R} k_{u}}\left\|\nabla\left(\bar{R} k_{u} u_{\varepsilon}-M\right)_{+}\right\|_{\Omega_{\varepsilon}, t}^{2} \\
& \quad+\frac{D_{s}}{l_{s}} \varepsilon^{3}\left\|\nabla_{\Gamma}\left(l_{s} s_{\varepsilon}-M\right)_{+}\right\|_{\Gamma_{\varepsilon}, t}^{2}+\left(1-\frac{1}{2 \lambda}\right) \varepsilon\left\|\left(\bar{R} k_{u} u_{\varepsilon}-M\right)_{+}-\left(l_{s} s_{\varepsilon}-M\right)_{+}\right\|_{\Gamma_{\varepsilon}, t}^{2} \\
& \quad \leq c_{1}-\frac{1}{\bar{R} k_{u}}\left(M k,\left(\bar{R} k_{u} u_{\varepsilon}-M\right)_{+}\right)_{\Omega_{\varepsilon}, t}-\frac{1}{l_{s}} \varepsilon\left\langle M k,\left(l_{s} s_{\varepsilon}-M\right)_{+}\right\rangle_{\Gamma_{\varepsilon}, t},
\end{aligned}
$$

where we choose $\lambda>\frac{1}{2}$. Now we distinguish two cases:

(a) Either $\bar{R} k_{u} u_{\varepsilon}-M \leq 0$ and $l_{s} s_{\varepsilon}-M \leq 0$ almost everywhere in $\Omega_{\varepsilon}$ and $\Gamma_{\varepsilon}$, respectively. Then, $u_{\varepsilon} \in L^{\infty}\left(\Omega_{\varepsilon}\right)$ and $s_{\varepsilon} \in L^{\infty}\left(\Gamma_{\varepsilon}\right)$ for almost every $t \in[0, T]$ and the assertion holds true.

(b) Or there exists $V \subset \Omega_{\varepsilon}$ (not a null set) with $\bar{R} k_{u} u_{\varepsilon}-M>0$ in $V$ or there exists $V \subset \Gamma_{\varepsilon}$ (not a null set) with $l_{s} s_{\varepsilon}-M>0$ in $V$. Then, we choose $k$ such that the right-hand side is smaller than or equal to zero and we conclude

$$
\begin{gathered}
\frac{1}{2 \bar{R} k_{u}}\left\|\left(\bar{R} k_{u} u_{\varepsilon}-M\right)_{+}\right\|_{\Omega_{\varepsilon}}^{2}+\frac{1}{2 l_{s}} \varepsilon\left\|\left(l_{s} s_{\varepsilon}-M\right)_{+}\right\|_{\Gamma_{\varepsilon}}^{2}+\frac{D_{u}}{\bar{R} k_{u}}\left\|\nabla\left(\bar{R} k_{u} u_{\varepsilon}-M\right)_{+}\right\|_{\Omega_{\varepsilon}, t}^{2} \\
\quad+\frac{D_{s}}{l_{s}} \varepsilon^{3}\left\|\nabla_{\Gamma}\left(l_{s} s_{\varepsilon}-M\right)_{+}\right\|_{\Gamma_{\varepsilon}, t}^{2}+c_{1} \varepsilon\left\|\left(\bar{R} k_{u} u_{\varepsilon}-M\right)_{+}-\left(l_{s} s_{\varepsilon}-M\right)_{+}\right\|_{\Gamma_{\varepsilon}, t}^{2} \leq 0 .
\end{gathered}
$$

Copyright $@$ by SIAM. Unauthorized reproduction of this article is prohibited. 
This yields $\bar{R} k_{u} u_{\varepsilon}-M<0$ and $l_{s} s_{\varepsilon}-M<0$ almost everywhere in $\Omega_{\varepsilon}$ and $\Gamma_{\varepsilon}$, respectively, and for almost every $t \in[0, T]$.

The proof for $v_{\varepsilon}$ and $w_{\varepsilon}$ is very similar. With corresponding estimates as before we get that $\bar{R} k_{v} v_{\varepsilon}-M \leq 0$ and $l_{w} w_{\varepsilon}-M \leq 0$ almost everywhere in $\Omega_{\varepsilon}$ and $\Gamma_{\varepsilon}$, respectively, and for almost every $t \in[0, T]$.

Next, we show that the time derivatives of $u_{\varepsilon}$ and $v_{\varepsilon}$ are elements of $H_{0}^{1}\left(\Omega_{\varepsilon}\right)^{\prime}$.

Lemma A.4 (time-estimation in $\left.\left(H_{0}^{1}\right)^{\prime}\right)$. There exists a $C>0$, independent of $\varepsilon$, such that

$$
\left\|\partial_{t} u_{\varepsilon}\right\|_{L^{2}\left([0, T], H_{0}^{1}\left(\Omega_{\varepsilon}\right)^{\prime}\right)}+\left\|\partial_{t} v_{\varepsilon}\right\|_{L^{2}\left([0, T], H_{0}^{1}\left(\Omega_{\varepsilon}\right)^{\prime}\right)}<C .
$$

Proof. We start by writing the $H_{0}^{1}\left(\Omega_{\varepsilon}\right)^{\prime}$-Norm in full for $\partial_{t} u_{\varepsilon}$. In the following we use that test functions $\varphi$ in $H_{0}^{1}\left(\Omega_{\varepsilon}\right)$ are zero on the boundary $\Gamma_{\varepsilon}$ :

$$
\begin{aligned}
\left\|\partial_{t} u_{\varepsilon}\right\|_{H_{0}^{1}\left(\Omega_{\varepsilon}\right)^{\prime}} & =\sup _{\varphi \in H_{0}^{1}\left(\Omega_{\varepsilon}\right),\|\varphi\|=1}\left(\partial_{t} u_{\varepsilon}, \varphi\right)_{H_{0}^{1}\left(\Omega_{\varepsilon}\right)^{\prime} \times H_{0}^{1}\left(\Omega_{\varepsilon}\right)} \\
= & \sup _{\varphi \in H_{0}^{1}\left(\Omega_{\varepsilon}\right),\|\varphi\|=1}(\left(-D_{u} \nabla u_{\varepsilon}, \nabla \varphi\right)_{H_{0}^{1}\left(\Omega_{\varepsilon}\right)^{\prime} \times H_{0}^{1}\left(\Omega_{\varepsilon}\right)}-\underbrace{\varepsilon\left\langle k_{u} R u_{\varepsilon}-l_{s} s_{\varepsilon}, \varphi\right\rangle_{\Gamma_{\varepsilon}}}_{=0} \\
& \left.-\left(f\left(u_{\varepsilon}\right), \varphi\right)_{H_{0}^{1}\left(\Omega_{\varepsilon}\right)^{\prime} \times H_{0}^{1}\left(\Omega_{\varepsilon}\right)}\right) \\
\leq & \sup _{\varphi \in H_{0}^{1}\left(\Omega_{\varepsilon}\right),\|\varphi\|=1}\left(c_{1}\left\|\nabla u_{\varepsilon}\right\|_{L^{2}\left(\Omega_{\varepsilon}\right)}\|\nabla \varphi\|_{L^{2}(\Omega)}+c_{2}\left\|f\left(u_{\varepsilon}\right)\right\|_{L^{2}\left(\Omega_{\varepsilon}\right)}\|\varphi\|_{L^{2}\left(\Omega_{\varepsilon}\right)}\right) \\
\leq & c_{1}\left(\left\|\nabla u_{\varepsilon}\right\|_{\Omega_{\varepsilon}}+\left\|f\left(u_{\varepsilon}\right)\right\|_{\Omega_{\varepsilon}}\right) .
\end{aligned}
$$

Integration with respect to time yields

$$
\left\|\partial_{t} u_{\varepsilon}\right\|_{L^{2}\left([0, T], H_{0}^{1}\left(\Omega_{\varepsilon}\right)^{\prime}\right)}^{2} \leq c_{1}\left(\left\|\nabla u_{\varepsilon}\right\|_{\Omega_{\varepsilon}, t}^{2}+\left\|f\left(u_{\varepsilon}\right)\right\|_{\Omega_{\varepsilon}, t}^{2}\right)<c_{2}
$$

where the boundedness holds because of Lemma A.2.

The proof for $\left\|\partial_{t} v_{\varepsilon}\right\|_{L^{2}\left([0, T], H_{0}^{1}\left(\Omega_{\varepsilon}\right)^{\prime}\right)}$ works analogously.

A.2. Proof of Lemma 4.3. We apply parts 2 and 3 of Lemma 2.4 to the weak equation of $s_{\varepsilon}$ and find

$$
\begin{aligned}
& \left(\partial_{t} \mathcal{T}_{\varepsilon}^{b}\left(s_{\varepsilon}\right), \psi\right)_{\Omega \times \Gamma}+D_{s}\left(\varepsilon \mathcal{T}_{\varepsilon}^{b}\left(\nabla_{\Gamma} s_{\varepsilon}\right), \nabla_{\Gamma} \psi\right)_{\Omega \times \Gamma} \\
& \quad=\left(k_{u} \mathcal{T}_{\varepsilon}^{b}\left(u_{\varepsilon}\right) \mathcal{T}_{\varepsilon}^{b}\left(R_{\varepsilon}\right)-l_{s} \mathcal{T}_{\varepsilon}^{b}\left(s_{\varepsilon}\right), \psi\right)_{\Omega \times \Gamma}-\left(\mathcal{T}_{\varepsilon}^{b}\left(h\left(s_{\varepsilon}\right)\right), \psi\right)_{\Omega \times \Gamma}
\end{aligned}
$$

for all $\psi \in L^{2}\left(\Omega, H_{\#}^{1}(\Gamma)\right)$. Now we write this equation for two epsilons $\varepsilon_{1}$ and $\varepsilon_{2}$ and subtract the equations from each other. As test function $\psi$ we take $\psi=\mathcal{T}_{\varepsilon_{1}}^{b}\left(s_{\varepsilon_{1}}\right)-$ $\mathcal{T}_{\varepsilon_{2}}^{b}\left(s_{\varepsilon_{2}}\right)$. Adding and subtracting the term $k_{u} \mathcal{T}_{\varepsilon_{2}}^{b}\left(u_{\varepsilon_{2}}\right) \mathcal{T}_{\varepsilon_{1}}^{b}\left(R_{\varepsilon_{1}}\right)$ yields

$$
\begin{aligned}
\left(\partial_{t}(\right. & \left.\left.\mathcal{T}_{\varepsilon_{1}}^{b}\left(s_{\varepsilon_{1}}\right)-\mathcal{T}_{\varepsilon_{2}}^{b}\left(s_{\varepsilon_{2}}\right)\right), \mathcal{T}_{\varepsilon_{1}}^{b}\left(s_{\varepsilon_{1}}\right)-\mathcal{T}_{\varepsilon_{2}}^{b}\left(s_{\varepsilon_{2}}\right)\right)_{\Omega \times \Gamma} \\
& +D_{s}\left\|\varepsilon_{1} \mathcal{T}_{\varepsilon_{1}}^{b}\left(\nabla_{\Gamma} s_{\varepsilon_{1}}\right)-\varepsilon_{2} \mathcal{T}_{\varepsilon_{2}}^{b}\left(\nabla_{\Gamma} s_{\varepsilon_{2}}\right)\right\|_{\Omega \times \Gamma}^{2}+l_{s}\left\|\mathcal{T}_{\varepsilon_{1}}^{b}\left(s_{\varepsilon_{1}}\right)-\mathcal{T}_{\varepsilon_{2}}^{b}\left(s_{\varepsilon_{2}}\right)\right\|_{\Omega \times \Gamma}^{2} \\
= & \left(k_{u} \mathcal{T}_{\varepsilon_{1}}^{b}\left(u_{\varepsilon_{1}}\right) \mathcal{T}_{\varepsilon_{1}}^{b}\left(R_{\varepsilon_{1}}\right)-k_{u} \mathcal{T}_{\varepsilon_{2}}^{b}\left(u_{\varepsilon_{2}}\right) \mathcal{T}_{\varepsilon_{1}}^{b}\left(R_{\varepsilon_{1}}\right)\right. \\
& \left.+k_{u} \mathcal{T}_{\varepsilon_{2}}^{b}\left(u_{\varepsilon_{2}}\right) \mathcal{T}_{\varepsilon_{1}}^{b}\left(R_{\varepsilon_{1}}\right)-k_{u} \mathcal{T}_{\varepsilon_{2}}^{b}\left(u_{\varepsilon_{2}}\right) \mathcal{T}_{\varepsilon_{2}}^{b}\left(R_{\varepsilon_{2}}\right), \mathcal{T}_{\varepsilon_{1}}^{b}\left(s_{\varepsilon_{1}}\right)-\mathcal{T}_{\varepsilon_{2}}^{b}\left(s_{\varepsilon_{2}}\right)\right)_{\Omega \times \Gamma} \\
& -\left(\mathcal{T}_{\varepsilon_{1}}^{b}\left(h\left(s_{\varepsilon_{1}}\right)\right)-\mathcal{T}_{\varepsilon_{2}}^{b}\left(h\left(s_{\varepsilon_{2}}\right)\right), \mathcal{T}_{\varepsilon_{1}}^{b}\left(s_{\varepsilon_{1}}\right)-\mathcal{T}_{\varepsilon_{2}}^{b}\left(s_{\varepsilon_{2}}\right)\right)_{\Omega \times \Gamma} \\
\leq & k_{u} \bar{R}\left\|\mathcal{T}_{\varepsilon_{1}}^{b}\left(u_{\varepsilon_{1}}\right)-\mathcal{T}_{\varepsilon_{2}}^{b}\left(u_{\varepsilon_{2}}\right)\right\|_{\Omega \times \Gamma}^{2}+k_{u} \bar{R}\left\|\mathcal{T}_{\varepsilon_{1}}^{b}\left(s_{\varepsilon_{1}}\right)-\mathcal{T}_{\varepsilon_{2}}^{b}\left(s_{\varepsilon_{2}}\right)\right\|_{\Omega \times \Gamma}^{2} \\
& +k_{u}\left\|u_{\varepsilon}\right\|_{L^{\infty}}\left\|\mathcal{T}_{\varepsilon_{1}}^{b}\left(R_{\varepsilon_{1}}\right)-\mathcal{T}_{\varepsilon_{2}}^{b}\left(R_{\varepsilon_{2}}\right)\right\|_{\Omega \times \Gamma}^{2} \\
& +k_{u}\left\|u_{\varepsilon}\right\|_{L^{\infty}}\left\|\mathcal{T}_{\varepsilon_{1}}^{b}\left(s_{\varepsilon_{1}}\right)-\mathcal{T}_{\varepsilon_{2}}^{b}\left(s_{\varepsilon_{2}}\right)\right\|_{\Omega \times \Gamma}^{2}+L_{h}\left\|\mathcal{T}_{\varepsilon_{1}}^{b}\left(s_{\varepsilon_{1}}\right)-\mathcal{T}_{\varepsilon_{2}}^{b}\left(s_{\varepsilon_{2}}\right)\right\|_{\Omega \times \Gamma}^{2} .
\end{aligned}
$$

Copyright (c) by SIAM. Unauthorized reproduction of this article is prohibited. 
Here we used that the function $h$ is Lipschitz-continuous with constant $L_{h}$. We integrate from 0 to $t$ and merge the constants to a single constant $c_{1}>0$,

$$
\begin{aligned}
&\left\|\mathcal{T}_{\varepsilon_{1}}^{b}\left(s_{\varepsilon_{1}}\right)-\mathcal{T}_{\varepsilon_{2}}^{b}\left(s_{\varepsilon_{2}}\right)\right\|_{\Omega \times \Gamma}^{2}+\left\|\varepsilon_{1} \mathcal{T}_{\varepsilon_{1}}^{b}\left(\nabla_{\Gamma} s_{\varepsilon_{1}}\right)-\varepsilon_{2} \mathcal{T}_{\varepsilon_{2}}^{b}\left(\nabla_{\Gamma} s_{\varepsilon_{2}}\right)\right\|_{\Omega \times \Gamma, t}^{2} \\
& \quad+\left\|\mathcal{T}_{\varepsilon_{1}}^{b}\left(s_{\varepsilon_{1}}\right)-\mathcal{T}_{\varepsilon_{2}}^{b}\left(s_{\varepsilon_{2}}\right)\right\|_{\Omega \times \Gamma, t}^{2} \\
& \leq c_{1}\left(\left\|\mathcal{T}_{\varepsilon_{1}}^{b}\left(s_{\varepsilon_{1}}\right)-\mathcal{T}_{\varepsilon_{2}}^{b}\left(s_{\varepsilon_{2}}\right)\right\|_{\Omega \times \Gamma, t}^{2}\right. \\
&\left.\quad+\left\|\mathcal{T}_{\varepsilon_{1}}^{b}\left(u_{\varepsilon_{1}}\right)-\mathcal{T}_{\varepsilon_{2}}^{b}\left(u_{\varepsilon_{2}}\right)\right\|_{\Omega \times \Gamma, t}^{2}+\left\|\mathcal{T}_{\varepsilon_{1}}^{b}\left(R_{\varepsilon_{1}}\right)-\mathcal{T}_{\varepsilon_{2}}^{b}\left(R_{\varepsilon_{2}}\right)\right\|_{\Omega \times \Gamma, t}^{2}\right) .
\end{aligned}
$$

With similar estimations we find

$$
\begin{aligned}
\| \mathcal{T}_{\varepsilon_{1}}^{b} & \left(R_{\varepsilon_{1}}\right)-\mathcal{T}_{\varepsilon_{2}}^{b}\left(R_{\varepsilon_{2}}\right) \|_{\Omega \times \Gamma}^{2} \\
\leq & c_{1}\left(\left\|\mathcal{T}_{\varepsilon_{1}}^{b}\left(R_{\varepsilon_{1}}\right)-\mathcal{T}_{\varepsilon_{2}}^{b}\left(R_{\varepsilon_{2}}\right)\right\|_{\Omega \times \Gamma, t}^{2}+\left\|\mathcal{T}_{\varepsilon_{1}}^{b}\left(s_{\varepsilon_{1}}\right)-\mathcal{T}_{\varepsilon_{2}}^{b}\left(s_{\varepsilon_{2}}\right)\right\|_{\Omega \times \Gamma, t}^{2}\right. \\
& +\left\|\mathcal{T}_{\varepsilon_{1}}^{b}\left(w_{\varepsilon_{1}}\right)-\mathcal{T}_{\varepsilon_{2}}^{b}\left(w_{\varepsilon_{2}}\right)\right\|_{\Omega \times \Gamma, t}^{2}+\left\|\mathcal{T}_{\varepsilon_{1}}^{b}\left(u_{\varepsilon_{1}}\right)-\mathcal{T}_{\varepsilon_{2}}^{b}\left(u_{\varepsilon_{2}}\right)\right\|_{\Omega \times \Gamma, t}^{2} \\
& \left.+\left\|\mathcal{T}_{\varepsilon_{1}}^{b}\left(v_{\varepsilon_{1}}\right)-\mathcal{T}_{\varepsilon_{2}}^{b}\left(v_{\varepsilon_{2}}\right)\right\|_{\Omega \times \Gamma, t}^{2}\right)
\end{aligned}
$$

and

$$
\begin{aligned}
&\left\|\mathcal{T}_{\varepsilon_{1}}^{b}\left(w_{\varepsilon_{1}}\right)-\mathcal{T}_{\varepsilon_{2}}^{b}\left(w_{\varepsilon_{2}}\right)\right\|_{\Omega \times \Gamma}^{2}+\left\|\varepsilon_{1} \mathcal{T}_{\varepsilon_{1}}^{b}\left(\nabla_{\Gamma} w_{\varepsilon_{1}}\right)-\varepsilon_{2} \mathcal{T}_{\varepsilon_{2}}^{b}\left(\nabla_{\Gamma} w_{\varepsilon_{2}}\right)\right\|_{\Omega \times \Gamma, t}^{2} \quad+\left\|\mathcal{T}_{\varepsilon_{1}}^{b}\left(w_{\varepsilon_{1}}\right)-\mathcal{T}_{\varepsilon_{2}}^{b}\left(w_{\varepsilon_{2}}\right)\right\|_{\Omega \times \Gamma, t}^{2} \\
& \leq c_{1}\left\|\mathcal{T}_{\varepsilon_{1}}^{b}\left(s_{\varepsilon_{1}}\right)-\mathcal{T}_{\varepsilon_{2}}^{b}\left(s_{\varepsilon_{2}}\right)\right\|_{\Omega \times \Gamma, t}^{2}+\left\|\mathcal{T}_{\varepsilon_{1}}^{b}\left(w_{\varepsilon_{1}}\right)-\mathcal{T}_{\varepsilon_{2}}^{b}\left(w_{\varepsilon_{2}}\right)\right\|_{\Omega \times \Gamma, t}^{2} \\
& \quad+\left\|\mathcal{T}_{\varepsilon_{1}}^{b}\left(v_{\varepsilon_{1}}\right)-\mathcal{T}_{\varepsilon_{2}}^{b}\left(v_{\varepsilon_{2}}\right)\right\|_{\Omega \times \Gamma, t}^{2}+\left\|\mathcal{T}_{\varepsilon_{1}}^{b}\left(R_{\varepsilon_{1}}\right)-\mathcal{T}_{\varepsilon_{2}}^{b}\left(R_{\varepsilon_{2}}\right)\right\|_{\Omega \times \Gamma, t}^{2} .
\end{aligned}
$$

Adding all three inequalities, using Gronwall's lemma and the trace inequality gives

$$
\begin{aligned}
\left\|\mathcal{T}_{\varepsilon_{1}}^{b}\left(s_{\varepsilon_{1}}\right)-\mathcal{T}_{\varepsilon_{2}}^{b}\left(s_{\varepsilon_{2}}\right)\right\|_{\Omega \times \Gamma}^{2}+\left\|\mathcal{T}_{\varepsilon_{1}}^{b}\left(w_{\varepsilon_{1}}\right)-\mathcal{T}_{\varepsilon_{2}}^{b}\left(w_{\varepsilon_{2}}\right)\right\|_{\Omega \times \Gamma}^{2}+\left\|\mathcal{T}_{\varepsilon_{1}}^{b}\left(R_{\varepsilon_{1}}\right)-\mathcal{T}_{\varepsilon_{2}}^{b}\left(R_{\varepsilon_{2}}\right)\right\|_{\Omega \times \Gamma}^{2} \\
\leq c_{1}\left(\left\|\mathcal{T}_{\varepsilon_{1}}^{b}\left(u_{\varepsilon_{1}}\right)-\mathcal{T}_{\varepsilon_{2}}^{b}\left(u_{\varepsilon_{2}}\right)\right\|_{\Omega \times \Gamma}^{2}+\left\|\mathcal{T}_{\varepsilon_{1}}^{b}\left(v_{\varepsilon_{1}}\right)-\mathcal{T}_{\varepsilon_{2}}^{b}\left(v_{\varepsilon_{2}}\right)\right\|_{\Omega \times \Gamma}^{2}\right) \\
\leq c_{1} c_{0}\left(\left\|\mathcal{T}_{\varepsilon_{1}}\left(u_{\varepsilon 1}\right)-\mathcal{T}_{\varepsilon_{2}}\left(u_{\varepsilon_{2}}\right)\right\|_{\Omega \times Y}^{2}+\left\|\varepsilon_{1} \mathcal{T}_{\varepsilon_{1}}\left(\nabla_{x} u_{\varepsilon 1}\right)-\varepsilon_{2} \mathcal{T}_{\varepsilon_{2}}\left(\nabla_{x} u_{\varepsilon_{2}}\right)\right\|_{\Omega \times Y}^{2}\right. \\
\left.\quad+\left\|\mathcal{T}_{\varepsilon_{1}}\left(v_{\varepsilon 1}\right)-\mathcal{T}_{\varepsilon_{2}}\left(v_{\varepsilon_{2}}\right)\right\|_{\Omega \times Y}^{2}+\left\|\varepsilon_{1} \mathcal{T}_{\varepsilon_{1}}\left(\nabla_{x} v_{\varepsilon 1}\right)-\varepsilon_{2} \mathcal{T}_{\varepsilon_{2}}\left(\nabla_{x} v_{\varepsilon_{2}}\right)\right\|_{\Omega \times Y}^{2}\right)
\end{aligned}
$$

where we used that $\nabla_{y} \mathcal{T}_{\varepsilon}\left(u_{\varepsilon}\right)=\varepsilon \mathcal{T}_{\varepsilon}\left(\nabla_{x} u_{\varepsilon}\right)$; see [9]. With integration with respect to time we find

$$
\begin{aligned}
& \left\|\mathcal{T}_{\varepsilon_{1}}^{b}\left(u_{\varepsilon_{1}}\right)-\mathcal{T}_{\varepsilon_{2}}^{b}\left(u_{\varepsilon_{2}}\right)\right\|_{\Omega \times \Gamma, t}^{2}+\left\|\mathcal{T}_{\varepsilon_{1}}^{b}\left(v_{\varepsilon_{1}}\right)-\mathcal{T}_{\varepsilon_{2}}^{b}\left(v_{\varepsilon_{2}}\right)\right\|_{\Omega \times \Gamma, t}^{2} \\
& \leq c_{1}|Y|(\underbrace{\left\|u_{\varepsilon_{1}}-u_{\varepsilon_{2}}\right\|_{\Omega, t}^{2}+\left\|v_{\varepsilon_{1}}-v_{\varepsilon_{2}}\right\|_{\Omega, t}^{2}}_{<\tilde{\delta}}
\end{aligned}
$$

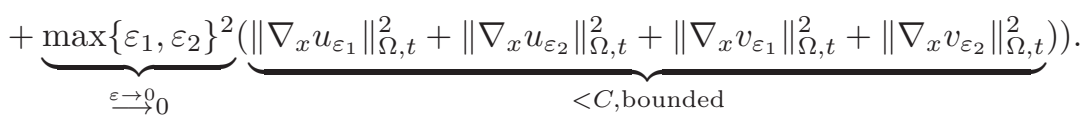

Because $u_{\varepsilon}$ and $v_{\varepsilon}$ converge strongly in $L^{2}([0, T] \times \Omega)$, there exists a $\tilde{\varepsilon}>0$ such that the first estimate holds true for $\varepsilon_{1}, \varepsilon_{2}<\tilde{\varepsilon}$. Hence, we deduce

$$
\begin{aligned}
& \left\|\mathcal{T}_{\varepsilon_{1}}^{b}\left(s_{\varepsilon_{1}}\right)-\mathcal{T}_{\varepsilon_{2}}^{b}\left(s_{\varepsilon_{2}}\right)\right\|_{\Omega \times \Gamma, t}^{2}+\left\|\mathcal{T}_{\varepsilon_{1}}^{b}\left(w_{\varepsilon_{1}}\right)-\mathcal{T}_{\varepsilon_{2}}^{b}\left(w_{\varepsilon_{2}}\right)\right\|_{\Omega \times \Gamma, t}^{2} \\
& \quad+\left\|\mathcal{T}_{\varepsilon_{1}}^{b}\left(R_{\varepsilon_{1}}\right)-\mathcal{T}_{\varepsilon_{2}}^{b}\left(R_{\varepsilon_{2}}\right)\right\|_{\Omega \times \Gamma, t}^{2} \leq c_{1}(\tilde{\delta}+\tilde{\varepsilon} C) \leq \delta
\end{aligned}
$$

Copyright (c) by SIAM. Unauthorized reproduction of this article is prohibited. 
for $\varepsilon_{1}, \varepsilon_{2}<\tilde{\varepsilon}$ and $\delta$ dependent on $\tilde{\varepsilon}$. This means that $s_{\varepsilon}, w_{\varepsilon}$, and $R_{\varepsilon}$ converge strongly in $L^{2}([0, T] \times \Omega \times \Gamma)$.

Appendix B. Existence of solutions of the microscopic problem: Proof of Theorem 4.4. We show existence of the solution $\left(u_{\varepsilon}, v_{\varepsilon}, s_{\varepsilon}, w_{\varepsilon}, R_{\varepsilon}\right)$ of the system of equations (3.2) for every $\varepsilon>0$. This is done in two steps. First we prove existence of the function $R_{\varepsilon} \in L^{2}\left([0, T] \times \Gamma_{\varepsilon}\right)$ when we assume the existence of $s_{\varepsilon}, w_{\varepsilon}, u_{\varepsilon}, v_{\varepsilon} \in$ $L^{2}\left([0, T] \times \Gamma_{\varepsilon}\right)$. Then, we show by using Schauder's theorem (see [29]) the existence of the solutions $u_{\varepsilon}, v_{\varepsilon}, s_{\varepsilon}$, and $w_{\varepsilon}$.

B.1. Existence for $\boldsymbol{R}_{\varepsilon}$. We start with considering the ordinary differential equation for $R_{\varepsilon}$ given in (3.1).

Lemma B.1 (existence of $R_{\varepsilon}$ ). Let $\varepsilon>0$ and $s_{\varepsilon}, w_{\varepsilon}, u_{\varepsilon}, v_{\varepsilon} \in L^{2}\left([0, T] \times \Gamma_{\varepsilon}\right)$. Then, there exists a solution $R_{\varepsilon} \in\left\{u \in L^{2}\left([0, T] \times \Gamma_{\varepsilon}\right) \mid \partial_{t} u \in L^{2}\left([0, T] \times \Gamma_{\varepsilon}\right)\right\}$ of the ordinary differential equation (3.1).

Proof. We use Carathéodory's existence theorem (see [10]). For this purpose, we define for almost every $x \in \Gamma_{\varepsilon}$ the function $j_{x}:[0, T] \times[0, \bar{R}] \rightarrow \mathbf{R}$ as

$$
j_{x}\left(t, R_{\varepsilon}\right):=-R_{\varepsilon}\left|k_{u} u_{\varepsilon}(t)+k_{v} v_{\varepsilon}(t)\right|+\left(\bar{R}-R_{\varepsilon}\right)\left|k_{s} s_{\varepsilon}(t)+k_{w} w_{\varepsilon}(t)\right| .
$$

Carathéodory's existence theorem states, if the following conditions hold for the function $j_{x}$ for almost every $x \in \Gamma_{\varepsilon}$, then there exists a solution $R_{\varepsilon}(\cdot, x) \in C([0, T])$ for almost every $x \in \Gamma_{\varepsilon}$ :

(a) The function $j_{x}$ is defined on a rectangle $[0, T] \times[0, \bar{R}]$.

(b) The function $j_{x}$ is measurable in $t$ for all fixed $R_{\varepsilon} \in[0, \bar{R}]$.

(c) The function $j_{x}$ is continuous in $R_{\varepsilon}$ for all fixed $t \in[0, T]$.

(d) There exists a Lebesgue-integrable function $m:[0, T] \rightarrow \mathbf{R}$ such that $\left|j_{x}\left(t, R_{\varepsilon}\right)\right| \leq m(t)$ for all $\left(t, R_{\varepsilon}\right) \in[0, T] \times[0, \bar{R}]$.

Conditions (a) and (c) are easily verified. Condition (b) is true because $u_{\varepsilon}, v_{\varepsilon}, s_{\varepsilon}, w_{\varepsilon}$ are $L^{2}$-functions and $|\cdot|$ is continuous. For $(\mathrm{d})$ we use that $u_{\varepsilon}(x), v_{\varepsilon}(x), s_{\varepsilon}(x)$, and $w_{\varepsilon}(x)$ are elements of $L^{2}([0, T])$ thus Lebesgue-integrable for almost every $x \in \Gamma_{\varepsilon}$. It follows that $j_{x}$ is Lebesgue-integrable itself and condition (d) is fulfilled. Hence, there exists a solution $R_{\varepsilon}(\cdot, x) \in C([0, T])$ for almost every $x \in L^{2}\left(\Gamma_{\varepsilon}\right)$.

We note that $R_{\varepsilon}(t, x) \in[0, \bar{R}]$ for almost every $t, x \in[0, T] \times \Gamma_{\varepsilon}$. The function $R:\left[L^{2}\left((0, T) \times \Gamma_{\varepsilon}\right)\right]^{4} \rightarrow C\left([0, T], L^{2}\left(\Gamma_{\varepsilon}\right)\right) \subset L^{2}\left([0, T] \times \Gamma_{\varepsilon}\right)$ with $R\left(u_{\varepsilon}, v_{\varepsilon}, s_{\varepsilon}, w_{\varepsilon}\right)=R_{\varepsilon}$ is bounded and continuous.

It is easily checked that the functions $f, g$, and $h$ are continuous and satisfy the growth condition $|\varphi(x)| \leq C|x|^{\frac{p}{q}}$ for $p=q=2$ and a $C>0$ for $\varphi=f, g$, and $h$. Then, with the theorem of Nemytskii (see [29]) it holds that the operators

$$
\begin{aligned}
F, G: L^{2}\left([0, T], L^{2}\left(\Omega_{\varepsilon}\right)\right) & \rightarrow L^{2}\left([0, T], L^{2}\left(\Omega_{\varepsilon}\right)\right), \\
F(u)(t) & =f(u(t)), \\
G(v)(t) & =g(v(t)), \\
& \text { and } \\
H: L^{2}\left([0, T], L^{2}\left(\Gamma_{\varepsilon}\right)\right) & \rightarrow L^{2}\left([0, T], L^{2}\left(\Gamma_{\varepsilon}\right)\right), \\
H(s)(t) & =h(s(t))
\end{aligned}
$$

are continuous and bounded for fixed $\varepsilon$.

B.2. Main part of the proof of Theorem 4.4. To complete the proof of the theorem, we use Schauder's theorem and Lemma B.1. We show that there exists a solution for a small time step $[0, \tau]$. To find the solution on the whole interval $[0, T]$ 
the solution parts must be linked together bit by bit. We define for a $\delta \in\left(0, \frac{1}{2}\right)$ the function space $V=L^{2}\left([0, \tau], H^{1-\delta}\left(\Omega_{\varepsilon}\right)\right)$ and $W:=L^{2}\left([0, \tau], L^{2}\left(\Gamma_{\varepsilon}\right)\right)$. Furthermore we define the mapping

$$
\begin{aligned}
S: V^{2} \times W^{2} \rightarrow & \left\{u \in L^{2}\left([0, \tau], H^{1}\left(\Omega_{\varepsilon}\right)\right) \mid \partial_{t} u \in L^{2}\left([0, \tau], H^{1}\left(\Omega_{\varepsilon}\right)^{\prime}\right)\right\}^{2} \\
& \times\left\{u \in L^{2}\left([0, \tau], H^{1}\left(\Gamma_{\varepsilon}\right)\right) \mid \partial_{t} u \in L^{2}\left([0, \tau], H^{1}\left(\Gamma_{\varepsilon}\right)^{\prime}\right)\right\}^{2}
\end{aligned}
$$

given by

$$
S\left(\hat{u}_{\varepsilon}, \hat{v}_{\varepsilon}, \hat{s}_{\varepsilon}, \hat{w}_{\varepsilon}\right)=\left(u_{\varepsilon}, v_{\varepsilon}, s_{\varepsilon}, w_{\varepsilon}\right)
$$

where $\left(u_{\varepsilon}, v_{\varepsilon}, s_{\varepsilon}, w_{\varepsilon}\right)$ is given by

$$
\begin{aligned}
\partial_{t} u_{\varepsilon}-D_{u} \Delta u_{\varepsilon} & =f\left(\hat{u}_{\varepsilon}\right) & & \text { in } \Omega_{\varepsilon}, \\
\partial_{t} v_{\varepsilon}-D_{v} \Delta v_{\varepsilon} & =g\left(\hat{v}_{\varepsilon}\right) & & \text { in } \Omega_{\varepsilon}, \\
-D_{u} \nabla u_{\varepsilon} \cdot n & =\varepsilon\left(k_{u} u_{\varepsilon} R\left(\hat{u}_{\varepsilon}, \hat{v}_{\varepsilon}, \hat{s}_{\varepsilon}, \hat{w}_{\varepsilon}\right)-l_{s} \hat{s}_{\varepsilon}\right) & & \text { on } \Gamma_{\varepsilon}, \\
-D_{v} \nabla v_{\varepsilon} \cdot n & =\varepsilon\left(k_{v} v_{\varepsilon} R\left(\hat{u}_{\varepsilon}, \hat{v}_{\varepsilon}, \hat{s}_{\varepsilon}, \hat{w}_{\varepsilon}\right)-l_{w} \hat{w}_{\varepsilon}\right) & & \text { on } \Gamma_{\varepsilon}, \\
\partial_{t} s_{\varepsilon}-\varepsilon^{2} D_{s} \Delta_{\Gamma} s_{\varepsilon} & =-l_{s} s_{\varepsilon}-h\left(\hat{s}_{\varepsilon}\right)+k_{u} u_{\varepsilon} R\left(\hat{u}_{\varepsilon}, \hat{v}_{\varepsilon}, \hat{s}_{\varepsilon}, \hat{w}_{\varepsilon}\right) & & \text { on } \Gamma_{\varepsilon}, \\
\partial_{t} w_{\varepsilon}-\varepsilon^{2} D_{w} \Delta_{\Gamma} w_{\varepsilon} & =-l_{w} w_{\varepsilon}+h\left(\hat{s}_{\varepsilon}\right)+k_{v} v_{\varepsilon} R\left(\hat{u}_{\varepsilon}, \hat{v}_{\varepsilon}, \hat{s}_{\varepsilon}, \hat{w}_{\varepsilon}\right) & & \text { on } \Gamma_{\varepsilon} .
\end{aligned}
$$

The system of partial differential equations (B.2) is linear and has a unique solution (see [12]) and the mapping $S$ is continuous. With the lemma of Lions-Aubin (see [29]) we know that $\left\{u \in L^{2}\left([0, \tau], H^{1}\left(\Omega_{\varepsilon}\right)\right) \mid \partial_{t} u \in L^{2}\left([0, \tau], H^{1}\left(\Omega_{\varepsilon}\right)^{\prime}\right)\right\}$ is compactly embedded in $V$ and $\left\{u \in L^{2}\left([0, \tau], H^{1}\left(\Gamma_{\varepsilon}\right)\right) \mid \partial_{t} u \in L^{2}\left([0, \tau], H^{1}\left(\Gamma_{\varepsilon}\right)^{\prime}\right)\right\}$ is compactly embedded in $W$. We deduce that the operator which maps $\left(\hat{u}_{\varepsilon}, \hat{v}_{\varepsilon}, \hat{s}_{\varepsilon}, \hat{w}_{\varepsilon}\right) \in V^{2} \times W^{2}$ to $\left(u_{\varepsilon}, v_{\varepsilon}, s_{\varepsilon}, w_{\varepsilon}\right) \in V^{2} \times W^{2}$ is continuous and compact.

Now, in order to apply Schauder's theorem, it is left to show that

$$
\begin{gathered}
\left(\left\|\hat{u}_{\varepsilon}\right\|_{V}^{2}+\left\|\hat{v}_{\varepsilon}\right\|_{V}^{2}+\left\|\hat{s}_{\varepsilon}\right\|_{W}^{2}+\left\|\hat{w}_{\varepsilon}\right\|_{W}^{2}\right) \leq r \quad \text { implies } \\
\left(\left\|u_{\varepsilon}\right\|_{V}^{2}+\left\|v_{\varepsilon}\right\|_{V}^{2}+\left\|s_{\varepsilon}\right\|_{W}^{2}+\left\|w_{\varepsilon}\right\|_{W}^{2}\right) \leq r
\end{gathered}
$$

for some $r>0$, where we may assume that the norms of the initial conditions are smaller than $r$. We test the equation for $u_{\varepsilon}$ of system (B.2) with $u_{\varepsilon}$ and integrate from 0 to $t<\tau$. Using standard estimations such as trace inequality, the Cauchy-Schwarz inequality, and the binomial theorem we find

$$
\begin{aligned}
\left\|u_{\varepsilon}\right\|_{\Omega_{\varepsilon}}^{2}+D_{u}\left\|\nabla u_{\varepsilon}\right\|_{\Omega_{\varepsilon}, t}^{2} & \leq c_{1}\left\|u_{\varepsilon}\right\|_{\Omega_{\varepsilon}, t}^{2}+\varepsilon^{2} c_{2}\left\|\nabla u_{\varepsilon}\right\|_{\Omega_{\varepsilon}, t}^{2}+c_{3} r \\
\left\|v_{\varepsilon}\right\|_{\Omega_{\varepsilon}}^{2}+D_{v}\left\|\nabla v_{\varepsilon}\right\|_{\Omega_{\varepsilon}, t}^{2} & \leq c_{1}\left\|v_{\varepsilon}\right\|_{\Omega_{\varepsilon}, t}^{2}+\varepsilon^{2} c_{2}\left\|\nabla v_{\varepsilon}\right\|_{\Omega_{\varepsilon}, t}^{2}+c_{3} r \\
\varepsilon\left\|s_{\varepsilon}\right\|_{\Gamma_{\varepsilon}}^{2}+\varepsilon^{3} D_{s}\left\|\nabla_{\Gamma} s_{\varepsilon}, t\right\|_{\Gamma_{\varepsilon}}^{2} & \leq c_{1} \varepsilon\left\|s_{\varepsilon}\right\|_{\Gamma_{\varepsilon}, t}^{2}+c_{2}\left\|u_{\varepsilon}\right\|_{\Omega_{\varepsilon}, t}^{2}+c_{3} \varepsilon^{2}\left\|\nabla u_{\varepsilon}\right\|_{\Omega_{\varepsilon}, t}^{2}+c_{4} r \\
\varepsilon\left\|w_{\varepsilon}\right\|_{\Gamma_{\varepsilon}}^{2}+\varepsilon^{3} D_{w}\left\|\nabla_{\Gamma} w_{\varepsilon}\right\|_{\Gamma_{\varepsilon}, t}^{2} & \leq c_{1} \varepsilon\left\|w_{\varepsilon}\right\|_{\Gamma_{\varepsilon}, t}^{2}+c_{2}\left\|v_{\varepsilon}\right\|_{\Omega_{\varepsilon}, t}^{2}+c_{3} \varepsilon^{2}\left\|\nabla v_{\varepsilon}\right\|_{\Omega_{\varepsilon}, t}^{2}+c_{4} r
\end{aligned}
$$

for some constants $c_{1}, c_{2}, c_{3}, c_{4}>0$. We add the results above and with $\varepsilon$ small we find

$$
\begin{aligned}
& \frac{1}{2}\left(\left\|u_{\varepsilon}\right\|_{\Omega_{\varepsilon}}^{2}+\left\|v_{\varepsilon}\right\|_{\Omega_{\varepsilon}}^{2}+\varepsilon\left\|s_{\varepsilon}\right\|_{\Gamma_{\varepsilon}}^{2}+\varepsilon\left\|w_{\varepsilon}\right\|_{\Gamma_{\varepsilon}}^{2}\right)+\left(D_{u}-\varepsilon^{2} c_{2}\right)\left\|\nabla u_{\varepsilon}\right\|_{\Omega_{\varepsilon}, t}^{2} \\
& \quad+\left(D_{v}-\varepsilon^{2} c_{2}\right)\left\|\nabla v_{\varepsilon}\right\|_{\Omega_{\varepsilon}, t}^{2}+\varepsilon^{3} D_{s}\left\|\nabla_{\Gamma} s_{\varepsilon}\right\|_{\Gamma_{\varepsilon}, t}^{2}+\varepsilon^{3} D_{s}\left\|\nabla_{\Gamma} w_{\varepsilon}\right\|_{\Gamma_{\varepsilon}, t}^{2} \\
& \leq c_{1}\left(\left\|u_{\varepsilon}\right\|_{\Omega_{\varepsilon}, t}^{2}+\left\|v_{\varepsilon}\right\|_{\Omega_{\varepsilon}, t}^{2}+\varepsilon\left\|s_{\varepsilon}\right\|_{\Gamma_{\varepsilon}, t}^{2}+\varepsilon\left\|w_{\varepsilon}\right\|_{\Gamma_{\varepsilon}, t}^{2}\right)+4 c_{4} r .
\end{aligned}
$$

Copyright (c) by SIAM. Unauthorized reproduction of this article is prohibited. 
With Gronwall's lemma we conclude

$$
\begin{aligned}
& \left\|u_{\varepsilon}\right\|_{\Omega_{\varepsilon}}^{2}+\left\|v_{\varepsilon}\right\|_{\Omega_{\varepsilon}}^{2}+\varepsilon\left\|s_{\varepsilon}\right\|_{\Gamma_{\varepsilon}}^{2}+\varepsilon\left\|w_{\varepsilon}\right\|_{\Gamma_{\varepsilon}}^{2} \\
& \quad+\left\|\nabla u_{\varepsilon}\right\|_{\Omega_{\varepsilon}, t}^{2}+\left\|\nabla v_{\varepsilon}\right\|_{\Omega_{\varepsilon}, t}^{2}+\varepsilon\left\|\nabla_{\Gamma} s_{\varepsilon}\right\|_{\Gamma_{\varepsilon}, t}^{2}+\varepsilon\left\|\nabla_{\Gamma} w_{\varepsilon}\right\|_{\Gamma_{\varepsilon}, t}^{2} \leq c_{1} r .
\end{aligned}
$$

This inequality (B.3) implies

$$
\begin{aligned}
& \left\|u_{\varepsilon}\right\|_{L^{2}\left((0, \tau), H^{1}\left(\Omega_{\varepsilon}\right)\right)}^{2}+\left\|v_{\varepsilon}\right\|_{L^{2}\left((0, \tau), H^{1}\left(\Omega_{\varepsilon}\right)\right)}^{2} \\
& \quad+\left\|s_{\varepsilon}\right\|_{L^{2}\left((0, \tau), H^{1}\left(\Gamma_{\varepsilon}\right)\right)}^{2}+\left\|w_{\varepsilon}\right\|_{L^{2}\left((0, \tau), H^{1}\left(\Gamma_{\varepsilon}\right)\right)}^{2} \leq c_{1} r .
\end{aligned}
$$

Integration from 0 to $\tau$ of inequality (B.3) gives

$$
\begin{aligned}
& \left\|u_{\varepsilon}\right\|_{L^{2}\left((0, \tau), L^{2}\left(\Omega_{\varepsilon}\right)\right)}^{2}+\left\|v_{\varepsilon}\right\|_{L^{2}\left((0, \tau), L^{2}\left(\Omega_{\varepsilon}\right)\right)}^{2} \\
& \quad+\varepsilon\left\|s_{\varepsilon}\right\|_{L^{2}\left((0, \tau), L^{2}\left(\Gamma_{\varepsilon}\right)\right)}^{2}+\varepsilon\left\|w_{\varepsilon}\right\|_{L^{2}\left((0, \tau), L^{2}\left(\Gamma_{\varepsilon}\right)\right)}^{2} \leq c_{1} r \tau .
\end{aligned}
$$

With the interpolation inequality (see [1])

$$
\|\cdot\|_{V} \leq \tilde{c}\|\cdot\|_{L^{2}\left((0, \tau), L^{2}\left(\Omega_{\varepsilon}\right)\right)}^{\delta}\|\cdot\|_{L^{2}\left((0, \tau), H^{1}\left(\Omega_{\varepsilon}\right)\right)}^{1-\delta}
$$

we get

$$
\left\|u_{\varepsilon}\right\|_{V}^{2}+\left\|v_{\varepsilon}\right\|_{V}^{2}+\left\|s_{\varepsilon}\right\|_{W}^{2}+\left\|w_{\varepsilon}\right\|_{W}^{2} \leq \tilde{c}\left(c_{1} r \tau\right)^{\delta}\left(c_{1} r\right)^{1-\delta} \leq r .
$$

The last inequality is correct if $\tau$ is chosen smaller than $\frac{1}{\left(\tilde{c} c_{1}\right)^{\frac{1}{\delta}}}$. Hence, the embedding composed with the mapping $S$ has at least one fixed point in $\left\{u \in L^{2}\left([0, \tau] ; H^{1}\left(\Omega_{\varepsilon}\right)\right) \mid\right.$ $\left.\partial_{t} u \in H^{1}\left(\Omega_{\varepsilon}\right)^{\prime}\right\}^{2} \times\left\{u \in L^{2}\left([0, \tau], H^{1}\left(\Gamma_{\varepsilon}\right)\right) \mid \partial_{t} u \in H^{1}\left(\Gamma_{\varepsilon}\right)^{\prime}\right\}^{2}$.

\section{REFERENCES}

[1] R. A. Adams and J. J. F. Fournier, Sobolev Spaces, 2nd ed., Academic Press, New York, 2003.

[2] G. Allaire, Homogenization and two-scale convergence, SIAM J. Math. Anal., 23 (1992), pp. $1482-1518$.

[3] A. Bensoussan, J.-L. Lions, and G. Papanicolaou, Asymptotic Analysis for Periodic Structures, North-Holland, Amsterdam, 1978.

[4] D. O. Besong, Mathematical Modelling and Numerical Solution of Chemical Reactions and Diffusion of Carcinogenic Compounds in Cells. M.S. thesis. KTH Numerical Analysis and Computer Science, 2004.

[5] D. Cioranescu, A. Damlamian, P. Donato, G. Griso, and R. Zaki, The periodic unfolding method in domains with holes, SIAM J. Math. Anal., 44 (2012), pp. 718-760.

[6] D. Cioranescu, A. Damlamian, and G. Griso, Periodic unfolding and homogenization, C. R. Math., 335 (2002), pp. 99-104.

[7] D. Cioranescu, A. Damlamian, and G. Griso, The periodic unfolding method in homogenization, SIAM J. Math. Anal., 40 (2008), pp. 1585-1620.

[8] D. Cioranescu and P. Donato, An Introduction to Homogenization, Oxford University Press, New York, 1999.

[9] D. Cioranescu, P. Donato, and R. Zaki, The periodic unfolding method in perforated domains, Port. Math., 63 (2006), pp. 467-496.

[10] E. A. Coddington and N. Levinson, Theory of Ordinary Differential Equations, McGrawHill, New York, 1955.

[11] A. M. Czochra and M. Ptashnyk, Derivation of a macroscopic receptor-based model using homogenization techniques, SIAM J. Math. Anal., 40 (2008), pp. 215-237.

[12] L. C. Evans, Partial Differential Equations, American Mathematical Society, Providence, RI, 2010.

[13] H. V. Gelboin, Benzo[a]parene metabolism, activation, and carcinogenesis: Role and regulation of mixed-function oxidases and related enzymes, Phys. Rev., 60 (1980), pp. 1107-1155.

Copyright (c) by SIAM. Unauthorized reproduction of this article is prohibited. 
[14] A. Gossauer, Struktur und Reaktivität der Biomoleküle, John Wiley \& Sons, New York, 2003.

[15] I. Graf And M. A. Peter, A convergence result for the periodic unfolding method related to fast diffusion on manifolds, C. R. Math., 352 (2014), pp. 485-490.

[16] I. Graf AND M. A. Peter, Homogenization of a carcinogenesis model with different scalings with the homogenization parameter, Math. Bohem., 139 (2014), pp. 163-184.

[17] I. Graf, M. A. Peter, AND J. Sneyd, Homogenization of a nonlinear multiscale model of calcium dynamics in biological cells, J. Math. Anal. Appl., 419 (2014), pp. 28-47.

[18] M. Höpker AND M. BöHm, A note on the existence of extension operators for Sobolev spaces on periodic domains, preprint, 2013.

[19] U. Hornung, Homogenization and Porous Media, Springer, New York, 1997.

[20] H. Jiang, S. L. Gelhaus, D. Mangal, R. G. Harvey, I. A. Blair, and T. M. Penning, Metabolism of benzo[a]pyrene in human bronchoalveolar h358 cells using liquid chromatography-mass spectrometry, Chem. Res. Toxicol., 20 (2007), pp. 1331-1341.

[21] V. V. Jikov, S. M. Kozlov, and O. A. Oleinik, Homongenization of Differential Operators and Integral Functionals, Springer, New York, 1994.

[22] V. A. Marchenko and E. Ya. Khruslov, Homogenization of Partial Differential Equations, Birkhäuser, Basel, 2006.

[23] M. Neuss-Radu, Some extensions of two-scale convergence, C. R. Acad. Sci. Paris, Ser. I, 322 (1996), pp. 899-904.

[24] N. Panasenko and N. S. Bakhvalov, Homogenization: Averaging Processes in Periodic Media: Mathematical Problems in the Mechanics of Composite Materials, Kluwer Academic, New York, 1989.

[25] O. Pelkonen and D. W. Nebert, Metabolism of polycyclic aromatic hydrocarbons: Etiologic role in carcinogenesis, Pharma. Rev., 34 (1982), pp. 189-212.

[26] M. A. Peter And M. Böhm, Different choises of scaling in homogenization of diffusion and interfacial exchange in a porous medium, Math. Methods Appl. Sci., 31 (2008), pp. 1257-1282.

[27] D. H. Phillips, Fifty years of benzo[a]pyrene, Nature, 303 (1983), pp. 468-472.

[28] E. Sanchez-Palencia, Non-homogeneous Media and Vibration Theory, Springer, New York, 1980.

[29] R. E. Showalter, Monotone Operators in Banach Space and Nonlinear Partial Differential Equations, American Mathematical Society, New York, 1997.

[30] P. Sims, P. L. Grover, A. Swaisland, K. Pal, And A. Hewer, Metabolic activation of benzo(a)pyrene proceeds by a diol-epoxide, Nature, 252 (1974), pp. 326-328.

[31] M. NEUSS-RADU UND W. JäGER, Effective transmission conditions for reaction-diffusion processes in domains separated by an interface, SIAM J. Math. Anal., 39 (2007), pp. 687-720.

Copyright $@$ by SIAM. Unauthorized reproduction of this article is prohibited. 